\title{
À propos des vestiges humains du Magdalénien du Sud-Ouest de la France : l'enfant inédit de l'abri Lafaye (Tarn et Garonne, France)
}

About Human Remains of the Magdalenian of the South-West of France: the unpublished child of the Abri Lafaye (Tarn and Garonne, France)

Mélie Le Roy et Dominique Henry-Gambier

\section{OpenEdition}

Journals

Édition électronique

URL : http://journals.openedition.org/paleo/3372

DOI : $10.4000 /$ paleo.3372

ISSN : 2101-0420

Éditeur

SAMRA

Édition imprimée

Date de publication : 30 décembre 2017

Pagination : 157-178

ISSN : 1145-3370

Référence électronique

Mélie Le Roy et Dominique Henry-Gambier, «À propos des vestiges humains du Magdalénien du SudOuest de la France : I'enfant inédit de l'abri Lafaye (Tarn et Garonne, France) », PALEO [En ligne], 28 | 2017, mis en ligne le 01 juin 2018, consulté le 07 juillet 2020. URL : http://journals.openedition.org/ paleo/3372 ; DOl : https://doi.org/10.4000/paleo.3372

\section{(c) (i) $\Theta$}

PALEO est mis à disposition selon les termes de la licence Creative Commons Attribution - Pas d'Utilisation Commerciale - Pas de Modification 4.0 International. 


\title{
À propos des vestiges humains du Magdalénien du Sud-Ouest de la France : l'enfant inédit de l'abri Lafaye (Tarn et Garonne, France)
}

\author{
Mélie LE ROY(a), Dominique HENRY-GAMBIER ${ }^{(b)}$
}

\begin{abstract}
Résumé : Les sépultures en contexte magdalénien sont très rares et seulement cinq sépultures datées du Magdalénien moyen sont connues ; parmi elles, la sépulture de l'abri Lafaye (Bruniquel, Tarn-et-Garonne) est la seule actuellement qui concerne un sujet immature. C'est aussi la seule sépulture double car l'enfant est associé à un individu adulte féminin. C'est donc l'unique enfant retrouvé en contexte sépulcral pour cette culture. L'Abri Lafaye fut découvert et fouillé dans les années 1860 par Victor Brun (Conservateur du Museum).

Du fait de l'attribution tardive de la sépulture au Magdalénien, l'essentiel des recherches a porté sur l'individu adulte et l'enfant a été laissé de côté. Perdu et oublié, il ne fut redécouvert que dans les années 1990 par l'une d'entre nous (D. Henry-Gambier) et Edmée Ladier dans le grenier du Muséum d'histoire naturelle de Montauban. Un inventaire ainsi qu'une description des vestiges de l'enfant, complétés par une estimation de l'âge au décès et de l'état sanitaire permettent de réaliser des comparaisons métriques et morphologiques avec un échantillon restreint d'individus immatures du Paléolithique supérieur et d'enfants plus récents, issus d'une collection médiévale (Cognac-Saint-Martin). Les résultats permettent sans surprise de confirmer une attribution à l'homme anatomiquement moderne (Homo sapiens sl.) de l'enfant et d'apporter des données inédites pour un individu relevant de la classe d'âge [1-4] ans pour la période du Magdalénien. Le mauvais état de conservation ne permet pas de discuter des causes de la mort, ni des liens de parenté avec l'individu adulte.
\end{abstract}

Mots-clés : Magdalénien, individu immature, Lafaye, biologie.

Abstract: About Human Remains of the Magdalenian of the South-West of France: the unpublished child of the Abri Lafaye (Tarn and Garonne, France). Graves in Magdalenian context are very rare randomly five burials dating from Middle Magdalenian are known; among them the burial of "I'Abri Lafaye" (Bruniquel, Tarn-et-Garonne) is the only one that concerns an immature individual. It is also the only known double burial, since the child is associated with an adult female individual. It is therefore the only child found in funerary context for this culture. "L'Abri Lafaye" was discovered and excavated in the 1860 s by Victor Brun (Curator of the Museum of Montauban).

The burial was lately attributed to the Magdalenian, therefore the main research focused on the adult individual and the child was not studied, Lost and forgotten, the immature individual was only rediscovered in the 1990s by Dominique Henry-Gambier and Edmée Ladier in the attic of the Museum of Natural History of Montauban. An inventory as well as a description of the remains of the child, supplemented by an estimation of the age at death and health status, permit to make metric and morphological comparisons with a small sample of immature individuals from the Upper Paleolithic and more recent children, from a medieval necropolis (Cognac-Saint-Martin).

The results confirm the modern like anatomy of the child and bring unpublished data for an individual aged between [14] years for the Magdalenian period. Yet, the poor state of conservation prevents any discussion of the causes of death or the ties of kinship with the adult.

Key-words: Magdalenian, immature individual, Lafaye, Biology.

(a) Archaeology and Paleocology, School of Natural and Built environment, Queen's University of Belfast. - melieleroy@ hotmail.fr (b) CNRS - Collaboratrice PACEA, UMR 5199 PACEA, Université de Bordeaux, Bâtiment B8, Allée Geoffroy Saint-Hilaire, CS 50023, 33615 Pessac cedex - d.gambier@pacea.u-bordeaux.fr 


\section{Introduction}

Cet article concerne l'étude anthropologique du squelette d'un jeune enfant magdalénien découvert dans l'abri Lafaye (Tarn et Garonne) au $19^{\mathrm{e}}$ siècle et resté en grande partie inédit.

L'abri Lafaye est l'un des cinq abris (Château, Montastruc, Gandil et Plantade) qui s'ouvrent au pied d'un escarpement calcaire dominant la rive gauche de l'Aveyron dans le Tarn et Garonne. Au-dessus de cette falaise s'élèvent le château et le village de Bruniquel.

Découverts et fouillés au $19^{\circ}$ siècle principalement par V. Brun, conservateur du Muséum d'Histoire naturelle de Montauban, ces abris ont livré une documentation importante sur les occupations magdaléniennes de la région et à ce titre représentent des sites majeurs pour le Magdalénien du sud-ouest de la France. L'abri Lafaye est le seul site magdalénien où deux squelettes humains, un adulte (Lafaye 24) et un individu immature (Lafaye $25^{1}$ ) ont été découverts. Ils ont été exhumés en mai 1864 par V. Brun dans la partie ouest de l'abri à environ $2,50 \mathrm{~m}$ de la paroi. Le remplissage initial comprenait plusieurs niveaux magdaléniens dont un ensemble Magdalénien moyen (Ladier et Welté 1991). Une datation C ${ }^{14}$ en SMA d'un fragment de côte du squelette de l'adulte (GifA 9504715290+-150 BP, soit 18 189-18 856 cal BP$^{2}$ ) indique un synchronisme avec la phase ancienne du Magdalénien moyen (Gambier et al. 2000). L'enfant était à côté de l'adulte et les informations indiquent une inhumation simultanée. L'adulte tardivement reconnu comme contemporain du Magdalénien (Guéret 1953) a fait l'objet d'analyses partielles depuis sa découverte (ex. Broca 1866 ; Hamy 1874 ; Guéret 1953 ; Genet-Varcin et Miquel 1967 ; Gambier 1992 ; Henry-Gambier et al. 2007 ; Villotte 2009). L'enfant, inédit, était jusqu'aux années quatre-vingtdix représenté par une mandibule et des dents isolées. Une série de vestiges crâniens et infracrâniens lui appartenant ont été retrouvés par $\mathrm{E}$. Ladier alors conservatrice du Muséum d'Histoire naturelle de Montauban et D. HenryGambier. Ce squelette quoique fragmentaire, est important car les restes d'enfants en contexte magdalénien sont rares, peu d'entre eux semblent avoir été inhumés ; leur biologie n'est pas connue (Henry-Gambier 2006 ; HenryGambier 2014). L'analyse de l'enfant de l'abri Lafaye vient combler en partie cette lacune.

\section{Matériel et méthodes}

L'inventaire des os, leur état de conservation et une analyse morphométrique et comparative constituent le corps de cet article. Les mesures ont été empruntées à R. Martin (Braüer 1988), d'autres sont plus spécifiques aux sujets immatures (Fazekas et Kosa 1978 ; Duday, Laubenheimer, Tillier 1995 ; Scheuer et Black 2000). Les variations anatomiques non métriques des dents permanentes ont été qualifiées selon les codes de Scott et Turner (1997), celles des dents déciduales selon ceux de
Hanihara (1961). L'usure dentaire a été appréciée en référence aux travaux de Smith (1984). L'âge au décès à partir de l'évolution de la denture a été déterminé en référence à la méthode de Moorrees, Fanning, Hunt (1963a et b) pour certaines des dents inférieures et des propositions d'Ubelaker (1978) pour les autres.

Les échantillons de comparaison dentaire utilisés pour le Paléolithique supérieur sont construits à partir de la base de données établie en 2014 par l'un d'entre nous (D. HenryGambier). Les dents usées ou trop altérées ont été exclues. Les données de comparaison des os fossiles proviennent de la littérature (Henry-Gambier 2001). La comparaison avec des populations plus récentes repose sur l'analyse de la collection de Cognac-Saint-Martin (Sansilbano-Collilieux 1993) et sur des données de la littérature (Scheuer et Black 2000).

$\mathrm{Au}$ vu de la taille des échantillons de comparaison paléolithique et médiéval, il a été jugé nécessaire d'uniquement effectuer des diagrammes en boîte pour l'étude comparative. Ainsi, de potentielles différences peuvent être observées malgré de nombreuses lacunes dans les données, dues à l'état de conservation et la rareté du matériel.

\section{Inventaire et état de conservation}

Lafaye 25 est représenté par cent quarante-six restes osseux et vingt-quatre vestiges dentaires isolés ou en place (tabl. 1).

La majorité des éléments crâniens ne sont pas identifiables avec précision en raison de leur taille comprise entre quelques $\mathrm{mm}^{2}$ et un $\mathrm{cm}^{2}$. L'os occipital et l'os temporal droits sont les mieux conservés (fig. 1). Une petite partie de l'os sphénoïde a aussi été identifiée. Les os de la face ne sont pas représentés bien que des dents supérieures soient présentes. La mandibule en plusieurs fragments est partiellement conservée. Parmi les vingt-quatre dents, quatorze déciduales et dix permanentes ont été identifiées.

Le squelette infracrânien très partiel comprend : une partie des os des mains, des vertèbres et des côtes ainsi que les clavicules. Des fragments d'os long ont été attribués aux radius, ulna et fibula avec un degré de certitude plus faible en raison de leur état de conservation. (fig. 2).

Les cassures sont caractéristiques de cassures survenues sur os sec. L'examen macroscopique des os ne montre aucun signe majeur de météorisation. Observée à la loupe binoculaire, la surface des os est marquée par des traces linéaires, un écaillage localisé de la corticale, peu important. Du sédiment fixé par des vernis et des consolidants ainsi que des traces de colle, de plâtre, de mastics ancien et récent, de produit de moulage, d'encre de chine et de graphite (crayon) et des traces d'outils de fouille sont présents. En revanche, aucune modification ancienne d'origine anthropique ou animale et aucune trace de colorant (type ocre) n’a été observée.

(1) Numérotation (Oakley et al. 1971).

(2) Date calibrée avec le logiciel Calib 7.02, courbe intcal13.14c (Reimer et al. 2013) 


\begin{tabular}{|c|c|c|c|c|c|c|}
\hline $\mathbf{N}^{\circ}$ & Région anatomique & Os/dent & Côté & SUP/INF & Rang & Conservation \\
\hline LF25-1 & Crâne & Os occipital (partie basilaire) & & & & COMP \\
\hline LF25-2 à 7 & Crâne & Os occipital (écaille) & & & & FRG \\
\hline LF25-8 & Crâne & Os temporal & D & & & FRG \\
\hline LF25-9 & Crâne & Os sphénoïde & & & & FRG \\
\hline LF25-10 à 40 & Crâne & Eléments de voûte crânienne & & & & FRG \\
\hline LF25-41(a) & Mandibule & Branche et corps & D & & & FRG \\
\hline LF25-41(b) & Dent en place & M1 & D & INF & & \\
\hline LF25-42 & Mandibule & Symphyse & & & & FRG \\
\hline LF25-43(a) & Mandibule & Corps & G & & & FRG \\
\hline LF25-43(b) & Dent déciduale en place & $\mathrm{dm} 2$ & G & INF & & \\
\hline LF25-44 & Dent déciduale isolée & di1 & D & SUP & & \\
\hline LF25-45 & Dent déciduale isolée & di1 & G & SUP & & \\
\hline LF25-46 & Dent déciduale isolée & di2 & D & SUP & & \\
\hline LF25-47 & Dent déciduale isolée & di2 & G & SUP & & \\
\hline LF25-48 & Dent déciduale isolée & $\mathrm{dm} 1$ & D & SUP & & \\
\hline LF25-49 & Dent déciduale isolée & $\mathrm{dm} 2$ & $\mathrm{D}$ & SUP & & \\
\hline LF25-50 & Dent déciduale isolée & $\mathrm{dm} 2$ & G & SUP & & \\
\hline LF25-51 & Dent déciduale isolée & $\mathrm{Di}$ & $\mathrm{D} / \mathrm{G}$ & INF & & \\
\hline LF25-52 & Dent déciduale isolée & $\mathrm{Di}$ & $\mathrm{D} / \mathrm{G}$ & INF & & \\
\hline LF25-53 & Dent déciduale isolée & dc & G & INF & & \\
\hline LF25-54 & Dent déciduale en place & $\mathrm{dm} 1$ & D & INF & & \\
\hline LF25-55 & Dent déciduale en place & $\mathrm{dm} 1$ & G & INF & & \\
\hline LF25-56 & Dent déciduale en place & $\mathrm{dm} 2$ & D & INF & & \\
\hline LF25-57 & Dent permanentes isolée & $\mathrm{C}$ & $\mathrm{D}$ & SUP & & \\
\hline LF25-58 & Dent permanentes isolée & M1 & D & SUP & & \\
\hline LF25-59 & Dent permanentes isolée & M1 & G & SUP & & \\
\hline LF25-60 & Dent permanentes isolée & 11 & D & INF & & \\
\hline LF25-61 & Dent permanentes isolée & I1 & G & INF & & \\
\hline LF25-62 & Dent permanentes isolée & 12 & D & INF & & \\
\hline LF25-63 & Dent permanentes isolée & 12 & G & INF & & \\
\hline LF25-64 & Dent permanentes isolée & C & D & INF & & \\
\hline LF25-65 & Dent permanentes isolée & C & G & INF & & \\
\hline LF25-66 à 67 & Ceinture & Clavicule & $D / G$ & & & FRG \\
\hline LF25-68-69 & Thorax & Côte & $D / G$ & & 1 & FRG \\
\hline LF25-70 & Thorax & Côte & G & & 2 & FRG \\
\hline LF25-71 à 86 & Thorax & Côte & $\mathrm{D} / \mathrm{G}$ & & $?$ & FRG \\
\hline LF25-87 à 90 & Colonne vertébrale & VC (corps) & & & $3,4,5,6,7$ & FRG \\
\hline LF25-91 à 98 & Colonne vertébrale & VC (arc) & $\mathrm{D} / \mathrm{G}$ & & $3,4,5,6,7$ & FRG \\
\hline LF25-99 & Colonne vertébrale & VTH (corps) & & & $?$ & FRG \\
\hline LF25-100 à 103 & Colonne vertébrale & VTH (arc) & $?$ & & $?$ & FRG \\
\hline LF25-104 à 122 & Colonne vertébrale & VTH-VL ? (arcs) & & & $?$ & FRG \\
\hline LF25-123 & Membre supérieur & Radius & $\mathrm{D}$ & & & FRG \\
\hline LF25-124 & Membre supérieur & Ulna & D & & & FRG \\
\hline LF25-125 à 131 & Membre supérieur & Métacarpien & $\mathrm{D} / \mathrm{G}$ ? & & $2,3,4,5 ?$ & FRG \\
\hline LF25-132 à 136 & Membre supérieur & Phalanges proximales & $\mathrm{D} / \mathrm{G}$ & & $2,3,4,5 ?$ & FRG \\
\hline LF25-137 à 139 & Membre supérieur & Phalange médiale & $D / G$ & & $2,3,4 ?$ & COMP \\
\hline LF25-140-141 & Membre supérieur & Phalange distale & $\mathrm{D} / \mathrm{G} ?$ & & $2,3,4 ?$ & COMP \\
\hline LF25-142-144 & Membre inférieur & Fibula & $\mathrm{D} / \mathrm{G} ?$ & & & FRG \\
\hline LF25-145-147 & Membres & Os long & $?$ & & & FRG \\
\hline
\end{tabular}

Tableau 1 - Abri Lafaye (Tarn et Garonne), inventaire et numérotation des os et des dents de Lafaye 25.

Table 1 - Abri Lafaye (Tarn et Garonne), inventory and numbering of bones and teeth of Lafaye 25. 



Figure 1 - Le crâne : de Lafaye 25. (1) Écaille occipitale ; (2) Pars basilaris ; (3) Os temporal (photo M. Le Roy).

Figure 1 - Bone fragments from the skull of Lafaye 25. (1) occipital bone fragments ; (2) Pars basilaris ; (3) temporal bone (photo M. Le Roy). 


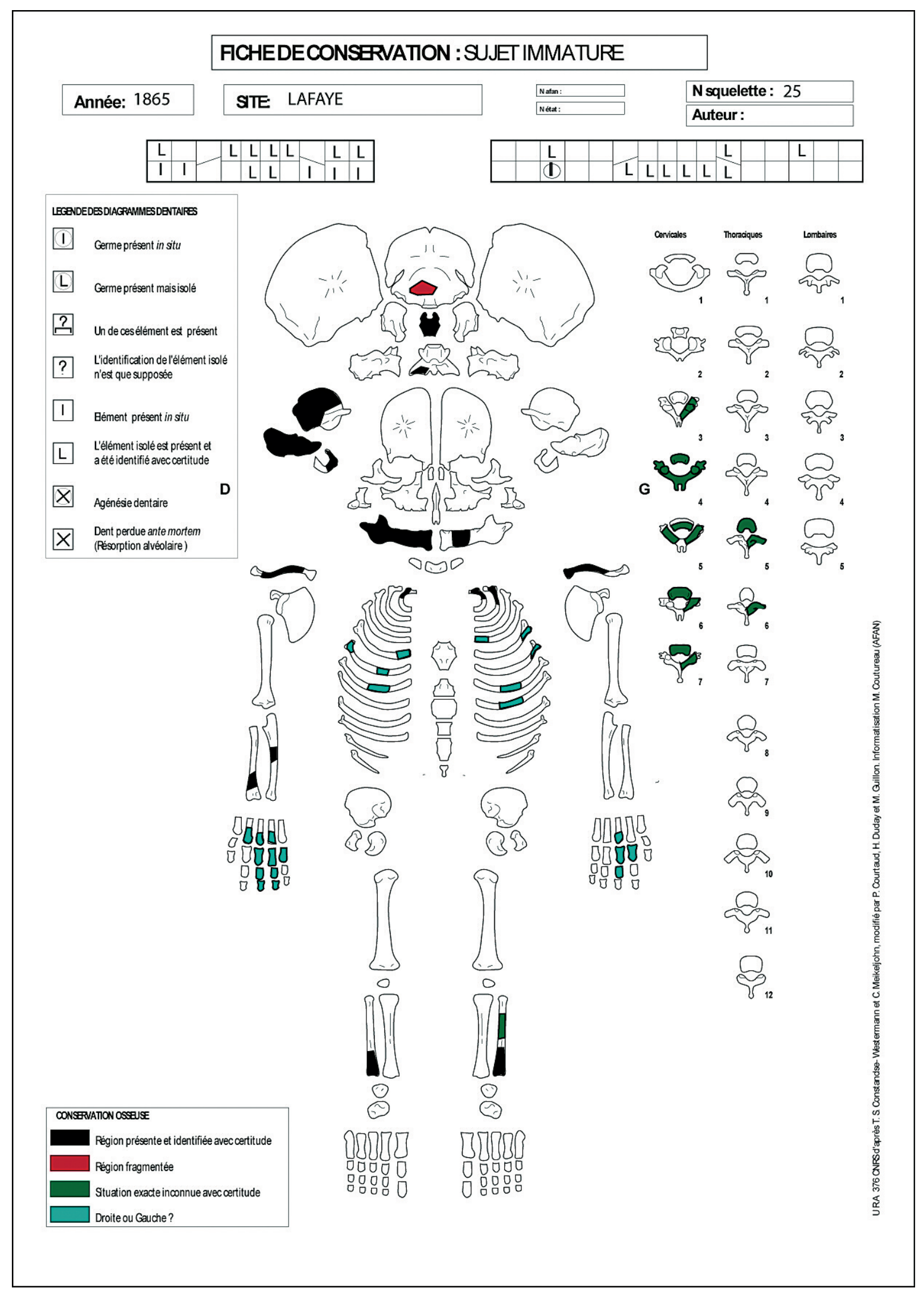

Figure 2 - Fiche de conservation des ossements de Lafaye 25.

Figure 2 - Preservative form of Lafaye 25. 
Certaines dents ont un émail altéré. Cette altération d'extension variable serait post-dépositionnelle selon C. Beauval (com. pers.). Les rares fissures longitudinales sur certaines dents et os sont probablement en relation avec la dessiccation postérieure à l'exhumation (séchage trop rapide) et peut-être variations de température et du degré d'hydrométrie importantes dans le lieu de conservation.

Le faible taux actuel de représentation du squelette (fig. 2) et l'état de surface des os et des dents s'expliquent par plusieurs facteurs : 1) la «brutalité » des fouilles (ex : la mandibule de l'enfant fut découverte à la suite d'un coup de pioche et la tête osseuse s'est désagrégée lors du prélèvement ; Brun 1866) ; 2) la méconnaissance des os d'enfants qui, en dépit du tamisage exceptionnel pour l'époque, a sans doute conduit au rejet et/ou à l'oubli d'os et/ou de dents ; 3) les aléas de la conservation et des restaurations.

Toutefois, l'état de surface des os collectés est comparable à celui des os de l'adulte et indique un enfouissement immédiat.

\section{1) Le crâne}

Du crâne, seuls l'occipital et l'écaille temporale droite se prêtent à une description.

L'os occipital est représenté par quatre éléments de l'écaille (fig.1) dont un, LF25-6, (long. : 29,8 mm) est bordé par un segment de la suture lambdoïde et par la partie basilaire. L'épaisseur de l'écaille varie de 1 à $2 \mathrm{~mm}$. Sur la face endocrânienne de l'écaille, lisse, s'ouvre un foramen dans la zone de la suture, distant de 2,2 $\mathrm{mm}$ du bord. Ce foramen ne débouche pas sur la face exocrânienne. L'épaisseur varie (médialement : 1,6 mm ; suture : 2,4 mm).

La partie basilaire de l'os occipital (LF25-1) est complète (fig. 1). Aucune trace de fusion avec les parties latérales (pars lateralis) et avec l'os sphénoïde, n'apparaît. Elle est plus large que longue (tabl. 2). La face supérieure est concave transversalement, de telle sorte que les surfaces de contact avec les parties latérales constituent des saillies à bord supérieur sinueux et aigu. Seule, une légère dépression y est observable, à la base du processus condylaire droit. La face inférieure est plane et de légers reliefs existent à l'emplacement du tubercule pharyngien chez l'adulte. Cette ébauche correspond chez l'homme actuel à un âge de 2 à 3 ans selon Scheuer et Black (2000). Les crêtes d'insertion du muscle petit droit antérieur commencent à s'individualiser. La surface de la partie basilaire des processus condylaires occipitaux lisse et allongée, forme une saillie vers le bas sur le bord postérieur. Une très faible asymétrie, le côté droit étant plus large que le gauche $(D=10,2 \mathrm{~mm} \mathrm{G}=9,1 \mathrm{~mm})$ est à noter. Le bord antérieur du foramen magnum est fin et régulier.

Les caractéristiques morphologiques de cet os sont analogues à celles d'autres jeunes enfants d'anatomie moderne fossiles ou récents de même classe d'âge (Scheuer et Black 2000 ; Henry-Gambier 2001). On note une forte variabilité de la robustesse de la Pars basilaris entre 1 et 5 ans (tabl. 2) tandis que ses proportions restent assez constantes (Scheuer et Black 2000). L'os de Lafaye 25 s'insère parmi les os les plus robustes.

L'os temporal droit (LF25-7) est incomplet $(27,3 \mathrm{~mm} / 69,6 \mathrm{~mm})$ et aplati (fig. 1). L'arcade zygomatique, ainsi que les régions antérieure et postérieure de l'écaille incluant la moitié postérieure du processus mastoïdien et les surfaces latérale et inférieure de l'os pétreux sont en partie détruites. La suture squamosale est présente sur 60 $\mathrm{mm}$. La hauteur, la longueur et la convexité de l'écaille ne sont pas mesurables. La face latérale de l'écaille n'offre aucune particularité. La partie supérieure de la suture pétrosquameuse subsiste sur $8 \mathrm{~mm}$. Cette suture est aussi visible sur la face médiale à la base de l'écaille. Le processus mastoïde commence à s'individualiser. L'incisure mastoïdienne est large et peu profonde. La face endocrânienne de l'os porte les empreintes des gyrus du lobe temporal. La région du méat auditif externe est trop partielle pour être décrite. Le bord supérieur (segment antérieur) de la partie pétreuse est marqué par un petit sillon $(2,9 \mathrm{~mm})$. L'éminence arquée et l'empreinte trigéminale sont bien modelées. La face postérieure montre le méat auditif interne (associé à la fossa subarcuata). II est de forme ovale et très profond. Une lame osseuse en divise le fond en deux parties inégales. En dépit des lacunes, la

\begin{tabular}{ccccccc}
\hline & $\begin{array}{c}\text { Long. } \\
\text { Maximale (2) }\end{array}$ & $\begin{array}{c}\text { Long.sagittale } \\
\text { (FK10 a)-(3) }\end{array}$ & $\begin{array}{c}\text { Larg.maximale } \\
\text { (FK10b)-(3) }\end{array}$ & $\begin{array}{c}\text { Haut. } \\
\text { synchondrose }\end{array}$ & $\begin{array}{c}\text { Larg. } \\
\text { synchondrose }\end{array}$ & Réf. \\
\hline Lafaye 25 [1-4] ans & 23,9 & 14,2 & 25,1 & 9,7 & 16,5 & $(1)$ \\
Grotte des Enfants 1 [2-4] ans & & 13,7 & 27,2 & $\mathrm{~nm}$ & $\mathrm{~nm}$ & $(1)$ \\
Arene Candide 5B [2-4] ans & & 14,5 & $\mathrm{~nm}$ & $\mathrm{~nm}$ & $\mathrm{~nm}$ & $(1)$ \\
Arene Candide 11 [2-4] ans & & 16,0 & 26,0 & $\mathrm{~nm}$ & $\mathrm{~nm}$ & $(1)$ \\
$\begin{array}{c}\text { Cognac-St-Martin [1-5] ans } \\
\text { (N=9) }\end{array}$ & $21,2-26,3$ & $14,2-19,4$ & $21,5-28$ & $9,4-11,4$ & $14,1-18,4$ \\
\hline
\end{tabular}

Tableau 2 - Abri Lafaye (Tarn et Garonne); dimensions de la pars basilaris de Lafaye 25 et des individus de comparaison. (1) : HenryGambier 2001; (2) : Scheuer et Black 2000; (3) : Fazekas et Kosa 1978.

Table 2 - Abri Lafaye (Tarn et Garonne); dimensions of the pars basilaris of Lafaye 25 and of the comparative sample individuals. HenryGambier 2001; (2) : Scheuer et Black 2000; (3) : Fazekas et Kosa 1978. 
fosse jugulaire allongée d'avant en arrière est visible.

Ce qui subsiste de cet os n'évoque pas des proportions ou une morphologie particulière par comparaison avec des enfants fossiles ou actuels d'anatomie moderne et de même classe d'âge.

L'os sphénoïde (LF25-9) est limité à un fragment de la petite aile droite (long. 29,5 mm), cassé sous le processus clinoïde antérieur. La face supérieure est lisse. Le bord postérieur est courbe et se termine normalement en arrière par le processus clinoïde antérieur. La vue inférieure fait apparaître une légère dépression. L'état de préservation ne permet pas de statuer sur la morphologie de l'os sphénoïde; aucun trait particulier ne semble cependant appréciable.

La mandibule est en deux parties (fig. 3), LF25-41a et 43a. Le premier (LF25-41a ; long.: 37,4 mm), comprend la branche droite dont le bord inférieur est érodé et une partie du corps droit avec le germe de M1 apparent par l'alvéole déhiscent. Le processus condylaire droit est complet. Le processus coronoïde et la lingula sont cassés. Les deux premières molaires déciduales ont été replacées sur l'arcade, en partie restaurée au plâtre ; la partie inférieure de la symphyse est présente; la partie alvéolaire est détruite. La jonction entre ces éléments est assurée par du plâtre de couleur claire. La radiographie montre que la restauration est très approximative (fig. 3 ). Le bord inférieur de la branche est souligné par un mastic bleu. La branche droite est basse et large (indice $=71,2$; tabl. 3). L'incisure mandibulaire est peu profonde, le bord de cette dernière est mince $(0,9 \mathrm{~mm})$. La face latérale, au niveau de l'incisure, présente une strie profonde et rectiligne (long. : 3,2 mm) due à un outil récent. Le processus condylaire est petit (larg. $=12,4 \mathrm{~mm}$ et épaisseur $=5,8 \mathrm{~mm}$; tabl. 3 ) et orienté transversalement, il se positionne au même niveau que le processus coronoïde. L'incisure sigmoïde le « coupe » de telle sorte que le tiers latéral apparaît comme le plus développé. Sur la face latérale de la branche on remarque quelques rugosités correspondant à l'insertion du muscle masséter. La ligne oblique n'est pas visible. Un renflement osseux est observable sur le corps de la mandibule à hauteur de la première molaire permanente. Le corps est lisse. Le foramen mandibulaire est profond et très évasé (long. : 5,8 mm ; haut. : 2,7 mm) avec au fond, plusieurs petits orifices séparés par des ponts osseux très fins. La ligne mylo-hyoïdienne est très peu marquée. Sous le processus condylaire, sur le bord médial de l'incisure mandibulaire, une fossette nette correspond vraisemblablement à l'insertion du muscle temporal. La seule observation possible sur le fragment symphysaire est la soudure effective des deux hémi mandibules.

Le second fragment de mandibule (LF25-43a) est une partie du corps gauche de l'alvéole de la deuxième molaire à celui de la canine déciduale (long. : $30,1 \mathrm{~mm}$ ). La hauteur $(15,6 \mathrm{~mm})$ et l'épaisseur $(7,7 \mathrm{~mm})$ mesurées au foramen mentonnier définissent un indice de robustesse de 50 (tabl. 3) qui indique un corps bas et moyennement épais et assez gracile (Braüer 1988). Le foramen mentonnier est situé sous la première molaire déciduale, position classique chez un enfant d'anatomie moderne. II est ovale (long. : 2,7 $\mathrm{mm}$; haut. : $1,9 \mathrm{~mm}$ ). Sur la face médiale, sous la première molaire déciduale gauche, le bord alvéolaire surplombe nettement le bord inférieur du corps. La fosse submandibulaire est très creusée et plusieurs foramens de petite taille s'y distinguent. L'extrémité antérieure de la ligne mylo-hyoïdienne est visible et normalement marquée.

Les régions mandibulaires conservées offrent une morphologie et des proportions conformes à celles d'enfant d'anatomie moderne fossile ou actuel d'âge comparable.

\section{2) Les dents (fig. 4 et tabl. 4)}

\section{Les dents déciduales supérieures (fig.4)}

L'incisive centrale droite (LF25-44) présente une couronne plus large que haute. Une légère altération de l'émail est visible au niveau du tubercule lingual. Le bord occlusal est usé (stade 4). La surface buccale ne présente pas de particularité. Le tubercule lingual et les crêtes distale et mésiale sont marqués : stade 1 (Hanihara 1961) équivalent au stade « effet en pelle ». L'apex de la racine est recourbé vers le côté buccal. L'incisive centrale gauche (LF25-45) dont l'apex de la racine est légèrement érodé est similaire à la droite. Les racines sont totalement calcifiées.

L'incisive latérale droite (LF25-46) a une couronne haute et étroite au bord incisif usé (stade 2). La racine est conservée sur les trois quarts de la hauteur initiale. Une grande partie des faces linguale et mésiale montrent une altération de l'émail (cf. supra). Le tubercule lingual est très prononcé. Les crêtes distale et mésiale sont bien marquées : stade 1 (Hanihara 1961). L'incisive latérale gauche (LF25-47), complète, est analogue à la dent symétrique.

La première molaire droite (LF25-48) présente une couronne allongée mésio-distalement et non usée. Les trois racines, divergentes, ne sont pas totalement calcifiées. Sur la face distale de la couronne, on retrouve l'altération de l'émail. Le renflement buccal à la base de la couronne est accusé. Les cuspides sont bien développées et les crêtes mésiale et distale sont nettes. Un sillon central forme une large dépression sur la surface occlusale. L'ensemble correspond à un stade $3 \mathrm{H} 2$ (Hanihara 1961).

La seconde molaire gauche (LF25-50) est représentée par la couronne non usée et trois racines incomplètement formées (fig. 4). La couronne est assez modelée avec un renflement au niveau du paracône. Un pont osseux reliant le métacône au protocône, sépare la fosse mésiale large et profonde, de la fosse distale, plus petite et moins profonde. Deux tubercules surnuméraires (tubercule du paracône mésial et protoconule) sont présents sur le bord mésial ; un métaconule existe sur le bord distal. Leur développement est de type stade 4 (Hanihara 1961). Un tubercule de Carabelli de stade 7 (Hanihara 1961) isolé par un sillon marqué est formé. La seconde molaire droite (LF25-49), similaire à la dent gauche, présente une altération de l'émail sur toute la surface occlusale et sur les faces mésiale et vestibulaire. Les racines prélevées pour l'analyse des isotopes (Drucker et al. en préparation) avaient un stade de calcification identique à celui observé sur la dent gauche. 

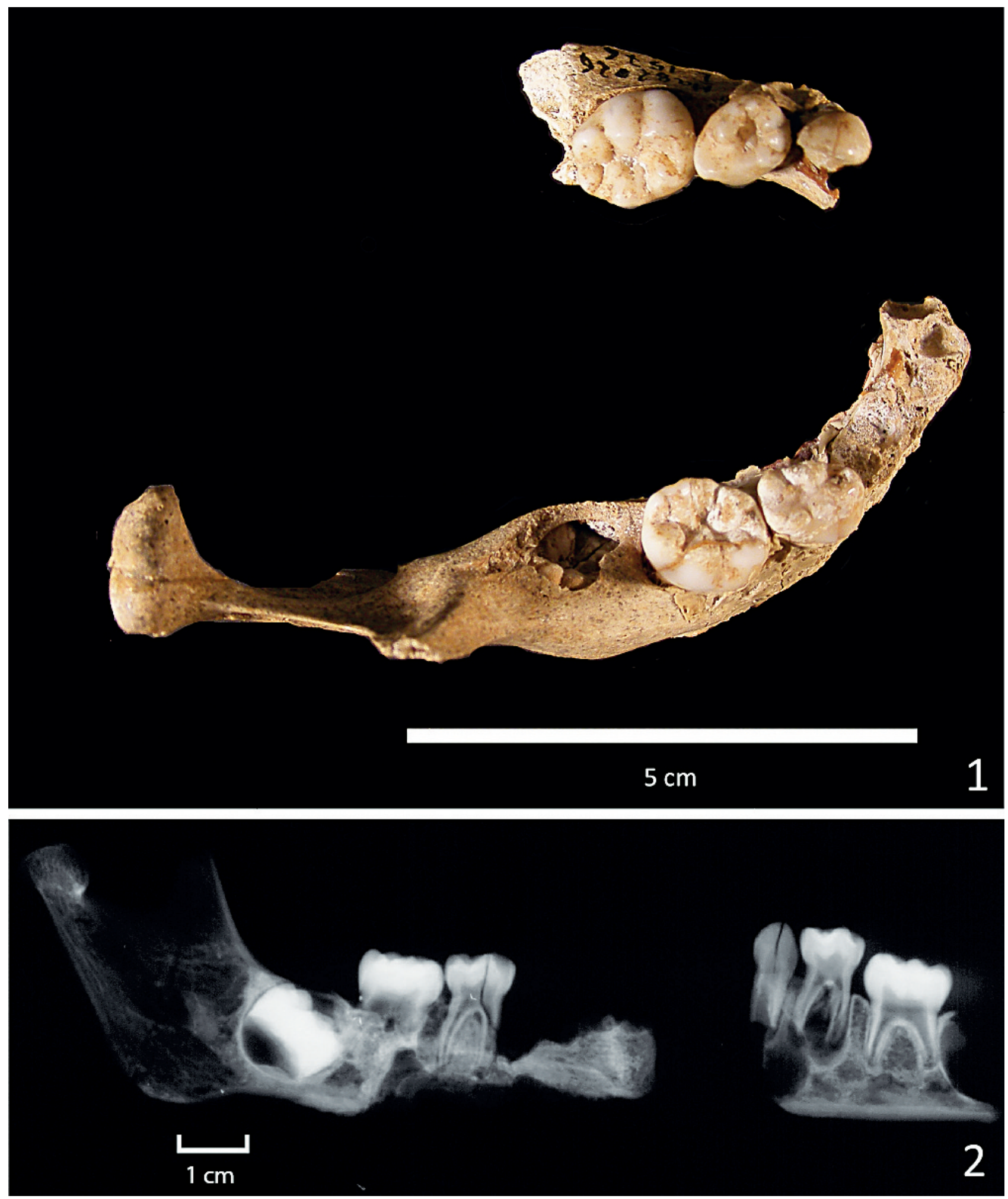

Figure 3 - Mandibule de Lafaye 25 vue occlusale (1) et radiographie (2), (photo M. Le Roy ; radio. M. Bessou, PACEA).

Figure 3 - Photograph from the occlusal view (1) and radiography (2) of the remains of the mandible of Lafaye 25 (photo M. Le Roy ; radio $M$. Bessou, PACEA). 


\begin{tabular}{|c|c|c|c|c|c|c|c|}
\hline & $\begin{array}{l}\text { Hauteur f. } \\
\text { mentonnier } \\
\text { G }\end{array}$ & $\begin{array}{c}\text { Epaisseur } \mathrm{f} \\
\text { mentonnier } \\
\mathrm{G}\end{array}$ & $\begin{array}{c}\text { Largeur } \\
\text { condyle } \\
\mathbf{G}\end{array}$ & $\begin{array}{l}\text { Epaisseur } \\
\text { condyle D }\end{array}$ & $\begin{array}{c}\text { Indice de } \\
\text { la } \\
\text { branche } \\
\text { D } \\
\end{array}$ & $\begin{array}{l}\text { Ind. de } \\
\text { robustesse } \\
\text { f. ment. G }\end{array}$ & Réf. \\
\hline Lafaye $25[1-4]$ ans & 15,6 & 7,7 & 12,4 & 5,8 & 71,2 & 50,0 & (1) \\
\hline Grotte des Enfants $1[2-4]$ ans & 17. 3 & 10. 9 & 14. 1 & 5. 6 * & 64,1 & 62. 9 & (1) \\
\hline Grotte des Enfants $2[2-4]$ ans & 17. 3 & 10. 5 & $\mathrm{~nm}$ & $\mathrm{~nm}$ & 77,4 & 60.5 & (1) \\
\hline Arene Candide 5B [2-4] ans & 17. 8 & 10. 0 & 12. 2 & 5. $7^{*}$ & $\mathrm{~nm}$ & 56. 2 & (1) \\
\hline Lafaye 24 adulte & $18^{*}$ & $10.7^{*}$ & $\mathrm{~nm}$ & $\mathrm{~nm}$ & 61,4 & $59.4^{*}$ & (4) \\
\hline $\begin{array}{c}\text { Cognac-St-Martin }[1-5] \text { ans } \\
\qquad(N=9)\end{array}$ & $14.5-21.4$ & $9.2-10.8$ & $10.8-13.1$ & $5.4-7.2$ & $56.5-90.3$ & $43.2-74.3$ & \\
\hline
\end{tabular}

Tableau 3 - Abri Lafaye (Tarn et Garonne); dimensions (en mm) de la mandibule. (1) : Henry-Gambier 2001 ; (4) Villotte 2009. Les dimensions suivies d'un * sont les mesures de l'élément anatomique symétrique (droit ou gauche).

Table 3 - Abri Lafaye (Tarn et Garonne); dimensions (mm) of the mandible. (1) : Henry-Gambier 2001 ; (4) Villotte 2009. The dimensions followed by * are from the symetric anatomical element (right or left).

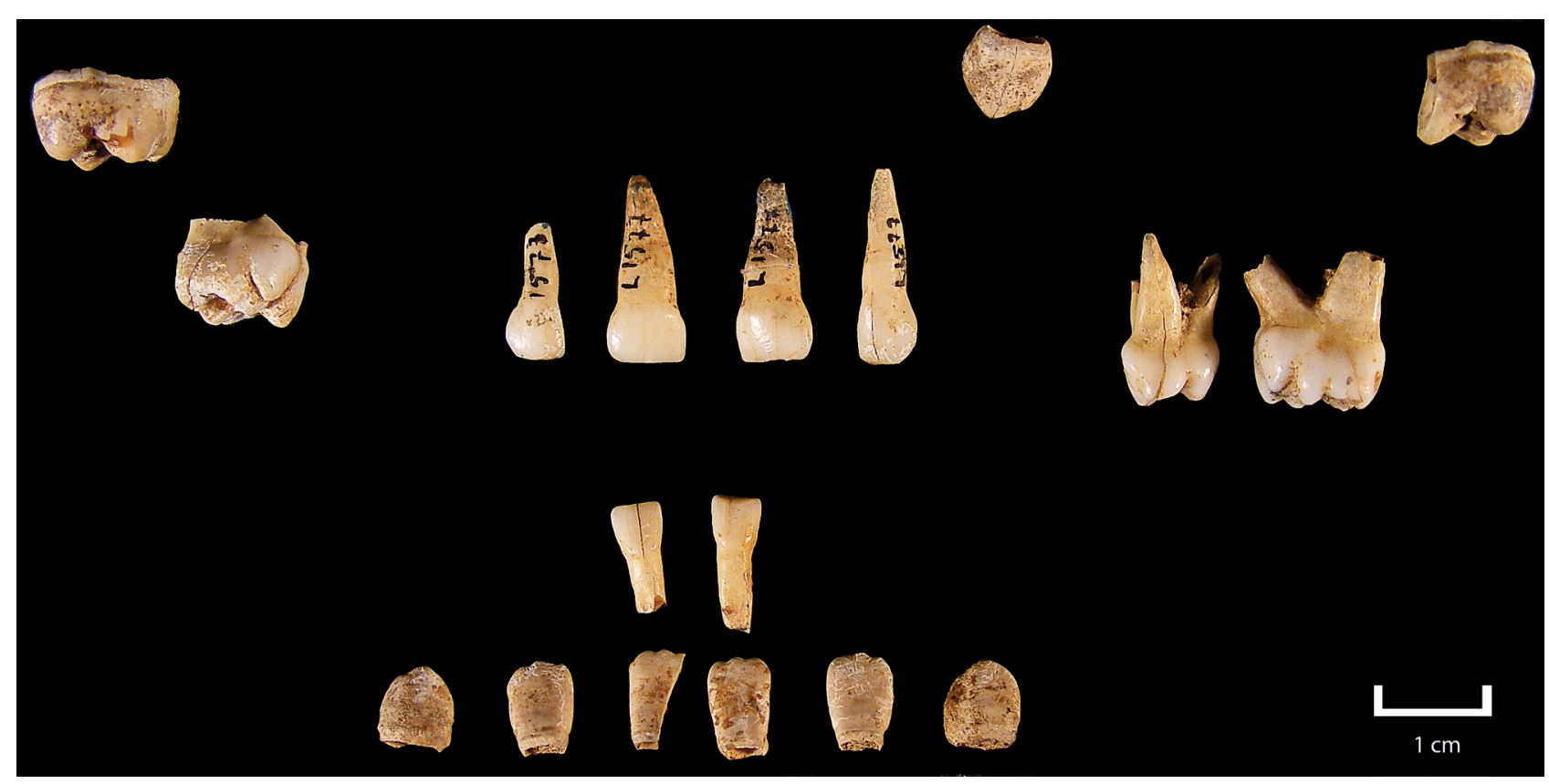

Figure 4 - Dents déciduales et permanentes isolées de Lafaye 25, face vestibulaire (photo M. Le Roy).

Figure 4 - Isolated decidual and permanent teeth from the vestibular view of Lafaye 25 (photo M. Le Roy).

\section{Les dents déciduales inférieures (fig. 4)}

L'incisive droite (LF25-51) présente une couronne étroite et sa racine n'est que partiellement conservée. Le bord incisif est usé (stade 3). Sur la face linguale, le tubercule est très légèrement développé. Les crêtes mésiale et distale sont peu marquées, stade 0 (Hanihara 1961). L'incisive gauche (LF25-52) est identique. L'étroitesse de la couronne (diamètre $\mathrm{MD}$ ) suggère un même rang et peut-être des incisives centrales.
La canine gauche (LF25-53) présente une racine en partie détruite. L'émail est altéré sur la face buccale. La couronne est asymétrique en raison d'un renflement à la base de la face buccale. Le tubercule lingual très développé, n'a aucun équivalent parmi les stades décrits par Hanihara (1961). Les crêtes mésiale et distale ne sont pas du tout marquées. L'usure est absente.

La première molaire gauche (LF25-54) comprend la couronne et les deux racines incomplètement formées. Elle est en place sur l'arcade (fig. 3). Des points d'altération de 


\begin{tabular}{|c|c|c|c|c|c|c|c|c|c|c|c|c|c|c|}
\hline & \multicolumn{14}{|c|}{ Dents supérieures déciduales } \\
\hline & \multicolumn{2}{|c|}{ dl1 D } & \multicolumn{2}{|c|}{ dl1 G } & \multicolumn{2}{|c|}{$\mathrm{dl} 2 \mathrm{D}$} & \multicolumn{2}{|c|}{$\mathrm{dl} 2 \mathrm{G}$} & \multicolumn{2}{|c|}{$\mathrm{dM} 1 \mathrm{D}$} & \multicolumn{2}{|c|}{$\mathrm{dM} 2 \mathrm{D}$} & \multicolumn{2}{|c|}{$\mathrm{dM} 2 \mathrm{G}$} \\
\hline & B-L & M-D & B-L & M-D & B-L & M-D & B-L & M-D & B-L & M-D & B-L & M-D & B-L & M-D \\
\hline Lafaye $25[1-4]$ ans & 5 & 6.3 & 4.8 & 6.4 & 4.2 & 4.5 & 4.1 & 4.7 & 5.8 & 8.6 & 8.4 & 10.3 & 9 & 10.4 \\
\hline $\begin{array}{c}\text { EUP-LUP [1-5] ans } \\
(\mathrm{N}=20)\end{array}$ & $\begin{array}{l}4.9- \\
6.1\end{array}$ & $\begin{array}{c}6.7- \\
8\end{array}$ & $\begin{array}{l}4.9- \\
5.8\end{array}$ & $\begin{array}{c}6.8- \\
8\end{array}$ & 5.1 & $\mathrm{~nm}$ & 5.1 & 5.1 & $\begin{array}{l}8.2- \\
10.4\end{array}$ & $\begin{array}{l}6.2- \\
8.6\end{array}$ & $\begin{array}{l}\text { 09- } \\
\text { nov }\end{array}$ & $\begin{array}{c}7.6- \\
10\end{array}$ & $\begin{array}{l}8.9- \\
11.3\end{array}$ & $\begin{array}{l}8.3- \\
10.5 \\
\end{array}$ \\
\hline \multirow[t]{4}{*}{$\begin{array}{l}\text { Cognac-St-Martin } \\
{[1-5] \text { ans }(N=16)}\end{array}$} & $\begin{array}{l}4.6- \\
5.8 \\
\end{array}$ & $\begin{array}{l}5.6- \\
6.3 \\
\end{array}$ & $\begin{array}{l}4.7- \\
5.6\end{array}$ & $\begin{array}{l}5.6- \\
6.5 \\
\end{array}$ & $\begin{array}{l}4.2- \\
5.1\end{array}$ & $\begin{array}{l}4.4- \\
5.3 \\
\end{array}$ & $\begin{array}{l}4.4- \\
5.3 \\
\end{array}$ & $\begin{array}{l}4.6- \\
5.4\end{array}$ & $\begin{array}{l}6.7- \\
8.6 \\
\end{array}$ & $\begin{array}{l}6.1- \\
9.1 \\
\end{array}$ & $\begin{array}{l}8.1- \\
9.7 \\
\end{array}$ & $\begin{array}{l}6.6- \\
9.1 \\
\end{array}$ & $\begin{array}{l}8.1- \\
9.9 \\
\end{array}$ & $\begin{array}{l}8.1- \\
10.1\end{array}$ \\
\hline & \multicolumn{14}{|c|}{ Dents supérieures permanentes } \\
\hline & \multicolumn{2}{|c|}{ CD } & \multicolumn{2}{|c|}{ M1 D } & \multicolumn{2}{|c|}{ M1 G } & & & & & & & & \\
\hline & B-L & M-D & B-L & M-D & B-L & M-D & & & & & & & & \\
\hline Lafaye $25[1-4]$ ans & 5.8 & 7.5 & $\mathrm{~nm}$ & 10.3 & 9.6 & 11.5 & & & & & & & & \\
\hline $\begin{array}{l}\text { EUP-LUP [1-5] ans } \\
(n=14)\end{array}$ & $\begin{array}{c}8.2- \\
9\end{array}$ & $\begin{array}{l}7.7- \\
8.2\end{array}$ & $\begin{array}{l}9.2- \\
13.4\end{array}$ & $\begin{array}{l}8.9- \\
11.7\end{array}$ & $\begin{array}{l}11.8- \\
13.4 \\
\end{array}$ & $\begin{array}{c}10- \\
12.8 \\
\end{array}$ & & & & & & & & \\
\hline Lafaye 24 adulte & 8.2 & 7.2 & 13.1 & 9.1 & 12.4 & 9.7 & & & & & & & & \\
\hline \multirow[t]{4}{*}{$\begin{array}{l}\text { Cognac-St-Martin } \\
{[1-5] \text { ans }(N=16)}\end{array}$} & 6.2 & 7.1 & $\begin{array}{l}7.1- \\
10.8\end{array}$ & $\begin{array}{l}9.5- \\
10.8 \\
\end{array}$ & $\begin{array}{l}6.3- \\
10.9 \\
\end{array}$ & 9.6 & & & & & & & & \\
\hline & \multicolumn{14}{|c|}{ Dents inférieures déciduales } \\
\hline & \multicolumn{2}{|c|}{ dl1 D } & \multicolumn{2}{|c|}{ dl1 G } & \multicolumn{2}{|c|}{$\mathrm{dC} \mathrm{G}$} & \multicolumn{2}{|c|}{ dM1 D } & \multicolumn{2}{|c|}{$\mathrm{dM} 1 \mathrm{G}$} & \multicolumn{2}{|c|}{$\mathrm{dM} 2 \mathrm{D}$} & \multicolumn{2}{|c|}{$\mathrm{dM} 2 \mathrm{G}$} \\
\hline & B-L & M-D & B-L & M-D & B-L & M-D & B-L & M-D & B-L & M-D & B-L & M-D & B-L & M-D \\
\hline Lafaye $25[1-4]$ ans & 3.5 & 3.9 & 3.7 & 3.8 & 5.1 & $\mathrm{~nm}$ & 7.6 & $\mathrm{~nm}$ & 8.3 & $\mathrm{~nm}$ & 9.2 & $\mathrm{~nm}$ & 8.9 & 10.5 \\
\hline $\begin{array}{c}\text { EUP-LUP [1-5] ans } \\
(\mathrm{N}=23)\end{array}$ & $\begin{array}{l}3.6- \\
4.2 \\
\end{array}$ & $\begin{array}{l}4.1- \\
4.8\end{array}$ & $\begin{array}{l}3.6- \\
5.2\end{array}$ & $\begin{array}{l}4.2- \\
6.9 \\
\end{array}$ & $\begin{array}{l}5.5- \\
6.3 \\
\end{array}$ & $\begin{array}{l}5.4- \\
7.3 \\
\end{array}$ & $\begin{array}{c}6.3- \\
8.1 \\
\end{array}$ & $\begin{array}{l}7.5- \\
9.5\end{array}$ & $\begin{array}{l}6.1- \\
8.6 \\
\end{array}$ & $\begin{array}{l}7.2- \\
9.3\end{array}$ & $\begin{array}{c}7.6- \\
11\end{array}$ & $\begin{array}{c}7- \\
11.7\end{array}$ & $\begin{array}{l}8.5- \\
10.5\end{array}$ & $\begin{array}{l}9.9- \\
11.2\end{array}$ \\
\hline \multirow[t]{4}{*}{$\begin{array}{l}\text { Cognac-St-Martin } \\
{[1-5] \text { ans }(N=16)}\end{array}$} & $\begin{array}{l}3.5- \\
4.6 \\
\end{array}$ & $\begin{array}{l}3.4- \\
4.9 \\
\end{array}$ & $\begin{array}{l}3.4- \\
4.2 \\
\end{array}$ & $\begin{array}{l}3.5- \\
4.1 \\
\end{array}$ & $\begin{array}{l}5.1- \\
5.8\end{array}$ & $\begin{array}{c}4.3- \\
6.5 \\
\end{array}$ & $\begin{array}{l}5.5- \\
7.1\end{array}$ & $\begin{array}{l}7.2- \\
8.4 \\
\end{array}$ & $\begin{array}{l}5.7- \\
6.6 \\
\end{array}$ & $\begin{array}{l}7.2- \\
8.5 \\
\end{array}$ & $\begin{array}{l}7.5- \\
8.3 \\
\end{array}$ & $\begin{array}{l}9.2- \\
10.4\end{array}$ & $\begin{array}{l}7.1- \\
8.5 \\
\end{array}$ & $\begin{array}{l}9.1- \\
10.1\end{array}$ \\
\hline & \multicolumn{14}{|c|}{ Dents inférieures permanentes } \\
\hline & \multicolumn{2}{|c|}{ I1 D } & \multicolumn{2}{|c|}{ I1 G } & \multicolumn{2}{|c|}{ I2 D } & \multicolumn{2}{|c|}{ I2 G } & \multicolumn{2}{|c|}{ CD } & & & & \\
\hline & B-L & M-D & B-L & M-D & B-L & M-D & B-L & M-D & B-L & M-D & B-L & M-D & B-L & M-D \\
\hline Lafaye 25 [1-4] ans & 5.5 & 4.7 & 5.2 & $\mathrm{~nm}$ & 5.2 & 5.5 & 5.3 & 4.9 & 5.3 & 6.1 & 5.3 & 6.5 & $\mathrm{~nm}$ & $\mathrm{~nm}$ \\
\hline $\begin{array}{c}\text { EUP-LUP [1-5] ans } \\
(n=21)\end{array}$ & $\begin{array}{l}6.1- \\
6.7 \\
\end{array}$ & $\begin{array}{l}5.3- \\
7.2 \\
\end{array}$ & $\begin{array}{l}6.1- \\
7.2 \\
\end{array}$ & $\begin{array}{l}5- \\
6.1 \\
\end{array}$ & $6.4-7$ & $\begin{array}{c}5.4- \\
7 \\
\end{array}$ & $\begin{array}{l}6.7- \\
7.5 \\
\end{array}$ & $\begin{array}{l}6.0- \\
7.0\end{array}$ & $\begin{array}{c}5.6- \\
9\end{array}$ & $\begin{array}{l}6.5- \\
7.8 \\
\end{array}$ & $\begin{array}{l}5.7- \\
9.2 \\
\end{array}$ & $\begin{array}{c}6.7- \\
8\end{array}$ & $\begin{array}{l}9.6- \\
11.6 \\
\end{array}$ & $\begin{array}{c}10.8- \\
13 \\
\end{array}$ \\
\hline Lafaye 24 adulte & 6.4 & 4.4 & 6.3 & 4.8 & 6.8 & 5.1 & 7 & 5 & 8.3 & 6.2 & 7.2 & 7.3 & 10.8 & 10.4 \\
\hline $\begin{array}{l}\text { Cognac-St-Martin } \\
{[1-5] \text { ans }(\mathrm{N}=16)}\end{array}$ & 4.3 & 4.7 & $\mathrm{~nm}$ & $\mathrm{~nm}$ & $\mathrm{Nm}$ & $\mathrm{nm}$ & $\mathrm{nm}$ & $\mathrm{nm}$ & $\mathrm{nm}$ & $\mathrm{nm}$ & $\mathrm{nm}$ & $\mathrm{nm}$ & $\begin{array}{l}9.4- \\
9.7\end{array}$ & $\begin{array}{l}10.4- \\
11.3 \\
\end{array}$ \\
\hline
\end{tabular}

Tableau 4 - Abri Lafaye (Tarn et Garonne); Diamètres $(\mathrm{mm})$ Bucco-lingual (B-L) et Mésio-distal (M-D) de Lafaye 25 et des individus de comparaison, mesures d'après (5) : Duday et al. 1995.

Table 4 - Abri Lafaye (Tarn et Garonne); Diameters ( $\mathrm{mm}$ ) Bucco-lingual (B-L) et Mesio-distal (M-D) of Lafaye 25 and of the comparative sample individuals, measurements from Duday et al. 1995.

l'émail existent sur la face mésiale. La bosse cervicale mésiale est marquée sur la face buccale. Les cinq cuspides (protocônide, métacônide, entocônide, hypocônide et hypoconulide) sont inégalement développées. Les deux plus importantes sont les protocônide et métacônide qui se rejoignent délimitant deux fosses, une mésiale et une distale plus profonde. Les trois cuspides distales, sont plus petites et séparées par des sillons. Aucun stade n'est défini par Hanihara (1961) pour les premières molaires déciduales. La première molaire droite (LF25-55) est similaire à la gauche (fig. 3). La couronne est caractérisée par une altération de l'émail.
La seconde molaire gauche (LF25-43) est en place sur l'arcade (fig. 3). Les racines sont incomplètement calcifiées. Les cinq cuspides classiques sont observables. L'hypoconulide est déporté du côté buccal. Une sixième cuspide (entoconulide ou protostylide) se développe du côté disto-lingual (stade 6, Hanihara 1961). Un sillon traverse la dent mésio-distalement. Son développement équivaut à un stade 1 (Hanihara 1961). On distingue une fovea antérieure. La dent symétrique (LF25-56) est analogue mais l'altération de l'émail est plus forte et les racines noyées dans le mastic semblent cassées (fig. 3). 


\section{Les dents permanentes supérieures (fig.4)}

La canine droite (LF25-57) à l'état de germe est limitée à la couronne incomplètement formée. Un renflement est visible du côté mésial. La face buccale est lisse. La face linguale porte des reliefs peu marqués. On discerne cependant les crêtes mésiale et distale. Six petits orifices formant un arc de cercle s'ouvrent sur la face mésiale. Une ligne d'hypoplasie énamélaire haute de $0,5 \mathrm{~mm}$ fait le tour de la couronne à $1,5 \mathrm{~mm}$ de la limite supérieure.

La première molaire gauche (LF25-58) est limitée à la couronne dont la calcification semble être achevée (fig. 4). Les cuspides (protocône, métacône, paracône et hypocône) séparées par des sillons profonds sont très arrondies. Un tubercule de Carabelli volumineux et isolé par un sillon marqué (stade 7, Scott et Turner 1997) est visible. A la base de la couronne existe une ligne d'hypoplasie de l'émail de $0,8 \mathrm{~mm}$ d'épaisseur située à $1,3 \mathrm{~mm}$ du bord supérieur de la couronne. La première molaire droite (LF25-59) est représentée par la moitié buccale de la couronne. La morphologie est analogue à celle de la dent symétrique Une ligne d'hypoplasie énamélaire de 0,3 mm distante de 1,3 $\mathrm{mm}$ du bord supérieur de la couronne est aussi visible.

\section{Les dents permanentes inférieures (fig.4)}

L'incisive centrale gauche (LF25-60) est représentée par la moitié distale de la couronne. A 1,5 mm de la base de la couronne, court une ligne d'hypoplasie de $0,4 \mathrm{~mm}$ de hauteur. Le bord incisif est légèrement crénelé. Aucun relief marqué ne caractérise les deux faces de la couronne.

Le germe de l'incisive latérale droite (LF25-61) est complet (fig. 4). Sur la face buccale, les crêtes mésiale et distale ainsi que le tubercule lingual sont peu marqués. A 1,3 mm de la base de la couronne, on retrouve la ligne d'hypoplasie énamélaire (hauteur : 0,5 mm). Les incisives latérales droite et gauche (LF25-62; 63) sont très semblables aux deux incisives centrales. La différence majeure réside dans un diamètre mésio-distal plus important (tabl. 4) et un tubercule lingual plus fortement marqué. La dent (LF25-62) a une ligne d'hypoplasie $(0,3 \mathrm{~mm}$ de hauteur) située à $1,8 \mathrm{~mm}$ de la limite inférieure de la couronne. La dent (LF25-63) en présente une de 0,4 mm d'épaisseur située à $1,5 \mathrm{~mm}$ de la limite de la couronne.

La canine droite (LF25-64) présente un angle mésial de la couronne arrondi (fig. 4). On observe du côté lingual l'ébauche des crêtes mésiale et distale. Une ligne d'hypoplasie nette $(0,5 \mathrm{~mm}$ d'épaisseur) est située à $1,7 \mathrm{~mm}$ de la limite inférieure de la couronne. La canine gauche (LF25-65) est identique à (LF25-64). La ligne d'hypoplasie mesure $0,4 \mathrm{~mm}$ d'épaisseur et est à $1,7 \mathrm{~mm}$ de la limite de la couronne. L'altération de l'émail n'existe pas sur la dent droite.

La première molaire droite (LF25-41a) est incomplètement formée (fig. 3). Seule la face occlusale se devine à travers l'alvéole déhiscent. Les cinq cuspides classiques sont observables.

\section{3) Le squelette infracrânien.}

La clavicule droite (LF25-66) est endommagée aux deux extrémités (long. 46,8 mm) mais l'extrémité acromiale n'est qu'érodée (fig. 5). La face supérieure lisse et convexe ne porte aucun relief. Sur la face antérieure, une dépression allongée à fond irrégulier est observable à hauteur de l'empreinte du muscle deltoïde. La circonférence au foramen, est de $15 \mathrm{~mm}$ (tabl. 5). L'amplitude de la double courbure n'est pas évaluable. La face inférieure plane présente plusieurs reliefs, notamment dans la zone qui, chez l'adulte, correspond à l'insertion du ligament conoïde, des muscles trapézoïde et deltoïde. Un foramen s'ouvre sous le sillon subclavier. Sur la clavicule gauche (LF25-67) l'extrémité acromiale manque (long. conservée : 54,8 mm) et l'extrémité sternale n'est qu'érodée (fig. 5). La circonférence au foramen est de $16 \mathrm{~mm}$ (tabl. 5). La face inférieure est plane. Les tubercules deltoïdien et conoïdien sont figurés par des rugosités. Un foramen nourricier est également visible sous le sillon subclavier. La face supérieure est lisse et convexe. Un second foramen s'ouvre à 10,7 mm de la cassure de l'extrémité acromiale. La présence d'une dépression allongée sur la face antérieure, comme sur l'os droit n'est pas vérifiable vu l'état de conservation.

Les côtes sont représentées par dix-neuf fragments. Les deux premières côtes et la deuxième côte gauche ont été identifiées. La taille des autres éléments ne permet pas la détermination du rang. Parmi eux deux extrémités sternales, trois extrémités vertébrales et quatre parties de corps ont été repérés. Sept autres fragments ne permettent aucune identification.

La première côte droite (LF25-68) est incomplète, il manque la partie sternale (long. : 26,8 mm). La face supérieure est rugueuse avec une légère dépression annonçant probablement les gouttières des côtes adultes. Une légère dépression existe à la base du tubercule. La face inférieure lisse, laisse voir la surface articulaire. Sur le col, en vue postérieure on observe une dépression à fond irrégulier. La première côte gauche (LF25-69) représentée par le tubercule et une partie du corps (long. : 23,6 mm) offre les mêmes caractéristiques que la droite.

La deuxième côte gauche (LF25-70) comprend l'extrémité vertébrale et une partie du corps attenant (long. : $36 \mathrm{~mm}$ ). La surface articulaire du col est suivie d'une profonde dépression à fond rugueux qui continue jusqu'à l'angle de la côte. La face supérieure présente plusieurs reliefs, ainsi qu'une crête peu marquée qui sépare le corps en deux parties (concave pour l'antérieure et convexe pour la postérieure) et pourrait annoncer l'insertion du muscle dentelé antérieur. La face inférieure est lisse et porte un foramen proche du bord postérieur, situé à $8,7 \mathrm{~mm}$ de la cassure.

La colonne vertébrale est représentée par trente-six fragments, dont douze attribuables aux vertèbres cervicales ( 4 corps et 8 hémi-arcs) et cinq aux vertèbres thoraciques ( 1 corps et 4 hémi-arcs). Les autres éléments (LF25-104-122) sont trop fragmentaires pour en déterminer le rang. Ni l'atlas et l'axis, ni les vertèbres lombaires n'ont pu être identifiés (fig. 5). 

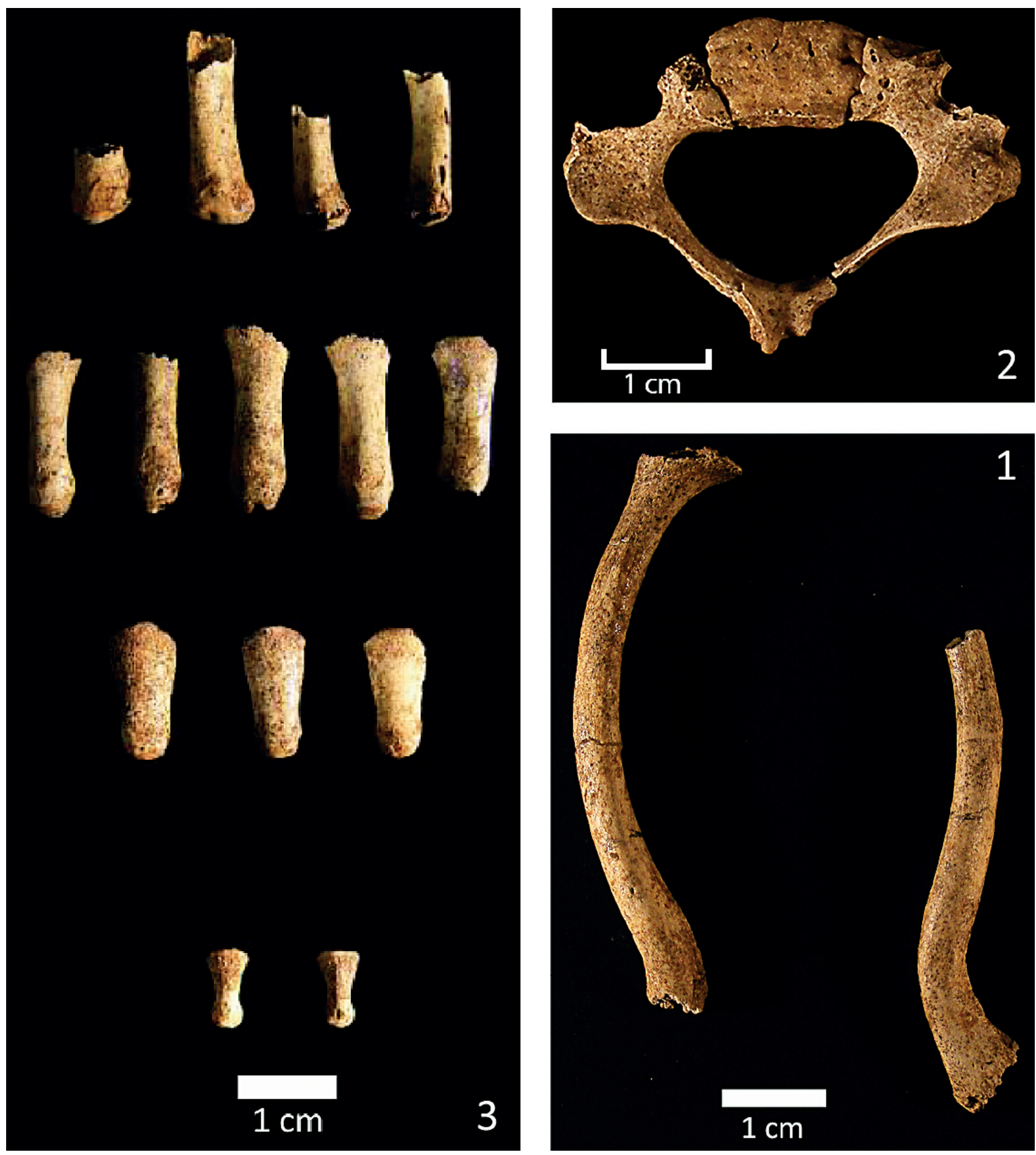

Figure 5 - Squelette infracrânien de Lafaye 25. (1) Clavicules ; (2) vertèbre ; (3) Métacarpiens et phalanges (photo M. Le Roy).

Figure 5 - infracranial skeletal elements of Lafaye 25. (1) Clavicles ; (2) vertebra ; (3) metacarpals and phalanges (photo M. Le Roy).

Quatre des cinq dernières vertèbres cervicales (C3 à $\mathrm{C} 7)$ sont représentées par le corps (LF25-87-90) bien conservé sauf celui de (LF25-89) qui est très incomplet. Plats et de forme trapézoïdale, ces corps présentent des sillons vasculaires sur les bords. Le bord antérieur est légèrement convexe et biseauté. Le bord postérieur est rectiligne. Sur
(LF25-89 ; 90) les bords latéraux font un angle et l'épaisseur est plus importante. Ils pourraient correspondre à C6 et C7. LF25-87 représenterait C3. Des orifices nombreux de taille variable s'ouvrent sur les différentes faces des corps. Les vertèbres cervicales sont aussi représentées par des hémi-arcs neuraux plus ou moins 


\begin{tabular}{ccccccccc}
\hline & \multicolumn{7}{l}{ long.max. clavicule (3) } & \multicolumn{2}{c}{ circonférence clavicule (5) } & indice robustesse (1) & Réf. \\
\hline & D & G & D & G & D & G \\
Lafaye 25 [1-4] ans & $69(46.8)$ & $69(54.9)$ & 15 & 16 & 21.7 & 23.2 & $(1)$ \\
Grotte des Enfants 1 [2-4] ans & 73.8 & 71.5 & 18 & 18 & 24.4 & 25.2 & $(1)$ \\
Grotte des Enfants 2 [2-4] ans & 69.2 & 68.1 & 17 & 16 & 24.6 & 23.5 & $(1)$ \\
Vado All' Arancio 2 & 61 & $\mathrm{~nm}$ & 17 & $\mathrm{~nm}$ & 27.9 & $\mathrm{~nm}$ & $(1)$ \\
Arene Candide 11 [2-4] ans & 70 & $\mathrm{~nm}$ & 15 & $\mathrm{~nm}$ & 21.4 & $\mathrm{~nm}$ & $(1)$ \\
Cognac-St-Martin [1-5] ans (N=7) & \multicolumn{2}{c}{$63.9-69.6$} & & $15-19$ & & $22.3-28.2$ & \\
\hline
\end{tabular}

Tableau 5 - Abri Lafaye (Tarn et Garonne); dimensions (en $\mathrm{mm}$ ) des longueurs ( ${ }^{*}$ est une valeur estimée, la longueur conservée est précisée entre parenthèse) des clavicules (3) : Fazekas et Kosa 1978, de la circonférence clavicule (5) : Duday et al. 1995 et de l'indice de robustesse (1) : Henry-Gambier 2001.

Table 5 - Abri Lafaye (Tarn et Garonne); Length dimensions ( $\mathrm{mm})$ (L ; Fazekas et Kosa 1978) and widths (I; Sladek et al. 2000) of the metacarpals (MTC3 and undetermined MTC) and of the proximal phalanges (MPP), medial (MPM) and distal (MPD) of Lafaye 25 and of the comparative sample individuals.

intacts. Un seul arc neural (LF25-93; 94) est presque entier. II est associé au corps (LF25-88) et pourrait concorder avec $\mathrm{C} 4$ ou $\mathrm{C} 5$, hypothèse que le processus épineux bifide et l'orientation des surfaces articulaires supérieures suggèrent. L'arc est fusionné et sa longueur maximum (Duday et al. 1995) est de $26,5 \mathrm{~mm}$. Les autres fragments d'arc montrent un redressement progressif des surfaces articulaires supérieures, normal sur la séquence C3-C7. L'hémi-arc (LF25-98) a une surface articulaire supérieure horizontale et plane compatible avec C3. Sur (LF25-96), les surfaces supérieures très redressées évoquent C7. Les autres fragments (LF25-91-92 ; 95 ; 97) ne présentent pas de particularités.

L'unique corps (LF25-30) de vertèbre thoracique est incomplet. Seul son bord postérieur est conservé, ainsi qu'une partie des bords latéraux, qui sont très divergents. Son épaisseur est supérieure à celle des corps des vertèbres cervicales $(5,8 \mathrm{~mm})$. II présente aussi des sillons vasculaires. Les hémi-arcs thoraciques (LF25-101-103) sont très fragmentaires et caractérisés par des surfaces articulaires très redressées, le rang n'est pas déterminable.

\section{Le membre supérieur}

Deux fragments (LF25-123) liés par un collage (long. : $39,3 \mathrm{~mm}$ ) ont une section triangulaire à base large et la portion conservée s'élargit vers l'une des extrémités. Cette morphologie évoque un fragment distal de radius (droit ?). La face antérieure est lisse et convexe. Le bord médial est saillant mais non aigu. La face postérieure est plane et lisse.

Un troisième fragment (LF25-124) conservé sur 25,4 mm serait compatible avec l'ulna. La section est triangulaire avec un bord très aigu. II présente une corticale épaisse et non régulière sur toute la circonférence. La diaphyse porte un foramen nourricier. Le foramen se situant sur la face antérieure et le bord le plus aigu étant latéral, il s'agirait donc d'un segment médian d'ulna droit. La face antérieure est lisse et plane. Sur la face opposée, une crête longitudinale divise la diaphyse en deux, une partie médiale concave et une latérale convexe.

Les mains sont représentées par dix-sept restes : sept métacarpiens dont trois très incomplets (LF25-125 à 131), cinq phalanges proximales, trois phalanges médiales et deux phalanges distales (fig. 5). Exceptées les phalanges distales et médiales, les autres os sont incomplets, leur rang et leur latéralisation sont donc très hypothétiques.

Le métacarpien (LF25-125) pourrait être le symétrique de (LF25-126) ce qui indiquerait que les mains droite et gauche sont représentées. Les extrémités distales des quatre métacarpiens les mieux conservés sont présentes. Elles sont globuleuses et ne permettent pas une identification précise ou la mise en évidence de caractères particuliers. À noter qu'aucun des fragments ne correspond au MTC I.

Quatre phalanges proximales (LF25 133-136) ont perdu l'extrémité proximale, une est intacte (LF25-132). La diaphyse est légèrement concave en face palmaire et convexe en face dorsale. On peut observer un foramen sur l'un des bords des diaphyses. Sa présence sur l'autre côté n'est certaine. L'extrémité distale est caractéristique d'une extrémité distale de phalange immature.

Rang et côté sont indéterminés sauf à noter l'absence de la phalange proximale du doigt 1 . Aucune symétrie n'est identifiable.

Trois phalanges médiales sont intactes. La hauteur de la diaphyse est peu importante (tabl. 6) ; un foramen s'observe sur un des côtés. Elles présentent une convexité dorsale et une concavité du côté palmaire. L'extrémité proximale offre une surface métaphysaire concave. L'extrémité distale est arrondie et les deux surfaces articulaires « débordent » latéralement et médialement sur la face palmaire. Aucun critère ne permet d'identifier d'éventuelles symétries. Ces phalanges médiales représentent les rayons 2 à 5 , mais il nous est impossible d'établir si les deux mains, droite et gauche, sont représentées. 


\begin{tabular}{lcccccccccc}
\hline & \multicolumn{2}{c}{ MTC3 } & \multicolumn{2}{c}{ MTC } & \multicolumn{2}{c}{ MPP } & \multicolumn{2}{c}{ MPM } & \multicolumn{2}{c}{ MPD } \\
\hline & long. & larg. & long. & larg. & long. & larg. & long. & larg. & long. & larg. \\
& $(3)$ & $(6)$ & $(3)$ & $(6)$ & $(3)$ & $(6)$ & $(3)$ & $(6)$ & $(3)$ & $(6)$ \\
& 20.8 & 4.7 & $13.4-$ & $3.1-$ & $16.5-$ & $4.6-$ & $13.4-$ & $5.1-$ & $8.1-$ & $2.7-$ \\
Lafaye 25 [1-4] ans & $21.4-$ & $4.3-$ & 16.7 & 4.3 & 18.9 & 5.3 & 13.8 & 5.8 & 8.2 & 2.8 \\
Cognac-St-Martin & $29.7-$ & $4.8-$ & $15.4-$ & $4.7-$ & $8.9-$ & $4.3-$ & $6.1-$ & $2-3.1$ \\
[1-5] ans (N=5) & 29.1 & 5.7 & 22.2 & 5.3 & 21.4 & 6.2 & 13.9 & 5.8 & 8.2 & $2-1$ \\
\hline
\end{tabular}

Tableau 6 - Abri Lafaye (Tarn et Garonne); dimensions (en mm) des longueurs (long. ; (3) : Fazekas et Kosa 1978) et largeurs (larg. ; (6) : Sladek et al. 2000) des métacarpes (MTC3 et MTC indéterminés) et des phalanges proximales (MPP), médiales (MPM) et distales (MPD) de Lafaye 25 et des individus de comparaison.

Table 6 - Abri Lafaye (Tarn et Garonne); Length dimensions ( $\mathrm{mm}$ ) (long. ; (3) : Fazekas et Kosa 1978) and widths (larg. ; (6) : Sladek et al. 2000) of metacarpals (MTC3 and undetermined MTC) and proximal phalanges (MPP), medial (MPM) and distal (MPD) of Lafaye 25 and of the comparative sample individuals.

Les deux phalanges distales sont complètes (LF25-140 ; 141). Elles ne peuvent être latéralisées et leur rang n'est pas déterminable. Une fois encore le doigt 1 et probablement le 5 ne sont pas représentés (tabl. 6).

\section{Le membre inférieur}

Parmi les fragments d'os longs, trois ont une morphologie suggérant une attribution à une fibula. Le fragment (LF25142) constitué d'éléments collés comprend les $2 / 3$ proximaux de la diaphyse (Long. : 47,4 mm). L'extrémité proximale est de forme ovale et plane. La diaphyse présente une section triangulaire, avec un bord osseux marqué sur une des faces. Ce bord délimite un côté concave, et un autre plus plat. Ce dernier est limité sur l'autre côté par une crête légèrement marquée.

Un second fragment conservé sur 31,6 mm (LF25-143) évoque aussi par son diamètre et sa morphologie une fibula.

Un troisième fragment (LF25-144) conservé sur 48,8 mm semble aussi compatible avec une fibula. La section de la diaphyse est triangulaire. Elle est marquée sur une face par un bord osseux divisant la diaphyse en deux parties, comme on a pu l'observer sur (LF25-142). Ce bord forme sur cette même face un méplat. Sur la face opposée, des bords interosseux sont visibles.

Aucun contact entre ces trois fragments n'a été mis en évidence. Les fragments (LF25-142 ; 144) représentant le même segment ; os droit et gauche seraient représentés. Un quatrième segment de diaphyse (LF25-145) long de $32,9 \mathrm{~mm}$ existe. La section est circulaire et la corticale est très épaisse. L'os a une forme évasée vers l'une de ses extrémités. Cependant l'absence de caractéristiques particulières (foramen, crête...), empêche une identification précise. L'épaisseur de la corticale serait compatible avec un humérus, un fémur ou un tibia humain mais un os de faune ne peut être écarté ${ }^{3}$.
Enfin aucune hypothèse sérieuse de détermination n'est formulable pour les fragments (LF25-146 ; 147).

\section{Résultats de l'analyse biologique et comparaisons}

\section{1) Âge au décès}

L'estimation de l'âge dentaire s'est heurtée à l'état des racines des dents souvent cassées ou érodées. À partir des dents déciduales inférieures, l'application de la méthode de Moorrees, Fanning, Hunt (1963 a et b) indique un âge dentaire compris entre 15 et 30 mois. À partir des dents permanentes inférieures, l'âge serait compris entre 27 et 48 mois. Selon les propositions de Ubelaker (1978), l'état de calcification de l'ensemble des dents correspondrait aux stades 2 ans +- 8 m et 3 ans +- 12 m. L'absence du germe de la première prémolaire permanente gauche (P3) inférieure ${ }^{4}$ et de toute ébauche de son alvéole est compatible avec l'estimation la plus basse de l'âge dentaire (ca. 15-24 mois). Sous la seconde molaire déciduale droite (dM2) et en avant de l'alvéole du germe de la première molaire permanente droite (M1), une «lacune » osseuse pourrait correspondre à l'alvéole de P3.

En raison de ces divers niveaux d'incertitude un âge dentaire compris entre [1-4] ans devrait être retenu, toutefois l'état de calcification des couronnes des dents permanentes suggère un âge se situant plutôt vers le haut de la fourchette.

La symphyse de la mandibule est complètement fusionnée. Ce processus prend place généralement autour de l'âge de 1 an (Scheuer et Black 2000). La suture intra-occipitale antérieure et la synchondrose sphéno-occipitale sont totalement ouvertes. La fusion de la première est en général complète vers 6 ans, celle de la seconde est plus tardive (Scheuer et Black 2000). Les arcs neuraux des vertèbres cervicales et thoraciques ne sont pas soudés aux corps. La seule vertèbre presque complète, une vertèbre

(3) L'identification des os longs a été discutée avec plusieurs faunistes dont C. Beauval.

(4) L'absence des germes des autres prémolaires n'est pas significative car l'état de conservation de la mandibule est compatible avec leur éventuelle disparition. 
cervicale de rang 4 ou 5, présente deux hémi-arcs neuraux soudés. Cette soudure intervient en général au cours de la seconde année (Scheuer et Black 2000). Un vestige de la suture petro-squameuse est visible sur la face latérale de l'os temporal droit et le processus mastoïde est peu développé ${ }^{5}$. In fine, les indices de maturation osseuse sont compatibles avec l'estimation proposée de l'âge dentaire. Les dimensions et proportions des autres éléments osseux (en particulier les clavicules) ne contredisent pas un âge au décès s'inscrivant dans l'intervalle [1-4] ans.

\section{2) Sexe}

Plusieurs méthodes de diagnose du sexe des sujets immatures existent à partir des os coxaux (Majò et al. 1993 ; Holcomb et Konisberg 1995), du crâne (Molleson et Cruse 1998 ; Loth et Henneberg 2001) ou des dimensions des couronnes dentaires (Hillson 1996 ; Saunders et al. 2007). La fiabilité de ces méthodes (Majò et al. 1993; Scheuer et Black 2000) restant très discutée et ici, leur mise en œuvre se heurtant au mauvais état de conservation du squelette (absence des os coxaux et crâne très incomplet), nous n'avons pas fait de tentative.

\section{3) État de santé et pathologie}

Aucune lésion osseuse ayant pu entraîner la mort n'a été observée. La seule caractéristique, en relation avec l'état de santé, voire les conditions de vie, est la présence d'hypoplasies de l'émail sur les germes des dents permanentes. Déficience dans la quantité et/ou l'épaisseur de l'émail dentaire (Hillson et Bond 1997), les hypoplasies illustreraient un épisode de stress survenu au cours du processus de calcification de la dent (Blakey et Armelagos 1985 ; Skinner and Goodman 1992). Des perturbations de la croissance (ex : fièvre, déficiences alimentaires) sont invoquées comme causes possible du stress (Goodman, Armelagos, Rose 1984 ; Skinner et Goodman 1992 ; Skinner 1996 ; Blakey et Armelagos 1997 ; Hillson et Bond 1997 ; Malville 1997 ; Reid et Dean 2000). Lorsque que plusieurs dents d'un même individu seraient concernées comme dans le cas de Lafaye 25, l'hypothèse d'un stress physiologique systémique est avancée (Guatelli-Steinberg, Buzhilova, Trinkaus 2011). L'état de conservation et de calcification des couronnes ne permet pas d'appliquer les méthodes d'estimation de l'âge de formation de ces défauts de l'émail (Goodman et Rose 1990 ; Malville 1997). Toutefois, la présence et la position des lignes d'hypoplasie sur les couronnes permanentes suggère au moins un épisode de stress alors que la couronne achève sa calcification, donc plus proche de la fin de vie du sujet que de la naissance. En outre, il est possible que les canines déciduales présentent sur la face vestibulaire une hypoplasie superficielle et très localisée de l'émail analogue à ce qui a été signalé sur les canines déciduales d'enfants épigravettiens (Skinner 1996 ; Henry-Gambier 20016). Le doute tient à l'extrême altération de la surface de la couronne. Ce type de défaut serait dû à un traumatisme mineur des améloblastes à travers une fenêtre dans l'os lorsque la dent est encore incluse. Les facteurs favorisants seraient la minceur de l'os qui serait liée à un trouble général d'ordre nutritionnel et les impacts qui se produiraient lorsque les enfants porteraient un objet à la bouche (Skinner et Hung 1989). Aux États-Unis, les groupes récents les plus atteints sont ceux où les enfants souffrent de malnutrition (Silberman 1989).

La radiographie des os longs ne révèle pas de ligne transversale (ligne de Harris) d'opacité plus marquée siégeant dans l'os spongieux diaphysaire ou juxtamétaphysaire (Wells 1967). Les os sont cependant très incomplets.

La présence de nombreux orifices vasculaires sur la face antérieure des corps vertébraux est en fait une variation classique, fréquemment rencontrée chez les enfants, ne signant pas une pathologie.

Enfin, aucun des os n'a de stigmate à mettre en relation avec des réactions périostées actives ou dépassées au moment du décès.

Un retard de croissance des prémolaires inférieures droites, voir une possible agénésie avait été envisagée (Henry-Gambier 2001). L'état de conservation de la mandibule dans la région des futures prémolaires ne permet pas de maintenir cette hypothèse.

\section{4) Analyse métrique et comparative}

\section{Dents déciduales}

La couronne des dents déciduales supérieures et inférieures de Lafaye 25 n'apparaît pas particulièrement volumineuse (tabl. 4) que l'on considère, l'échantillon des dents du début du Paléolithique supérieur (EUP) ou celui de la seconde moitié (LUP)7. Les diamètres bucco-linguaux et mésio-distaux (BL et MD) s'inscrivent dans la variation des deux échantillons, plus souvent du côté des valeurs basses (tabl. 4 ; fig. 6).

La comparaison avec les dents d'enfants d'une population médiévale, dont l'âge se situe entre 1 et 5 ans, montre que les dimensions dentaires de Lafaye 25 ne sortent pas de la variabilité et se situent majoritairement parmi les valeurs faibles (tabl. 4 ; fig. 6).

(5) Le développement de l'anneau tympanique ne peut être apprécié en raison de l'état de conservation de la région correspondante.

(6) Dans son article de 1996, M. Skinner, présente un inventaire des fossiles du Paléolithique supérieur européen montrant des hypoplasies de l'émail dentaire. Il faut remarquer que son échantillon sans doute établi à partir de documents anciens, comporte plus de $30 \%$ de sujets n'appartenant pas au Paléolithique supérieur mais à des périodes plus récentes.

(7) Données issues de la base de données inédite de D. Henry-Gambier - version 2 avril 2014. 
Canine déciduale inférieure

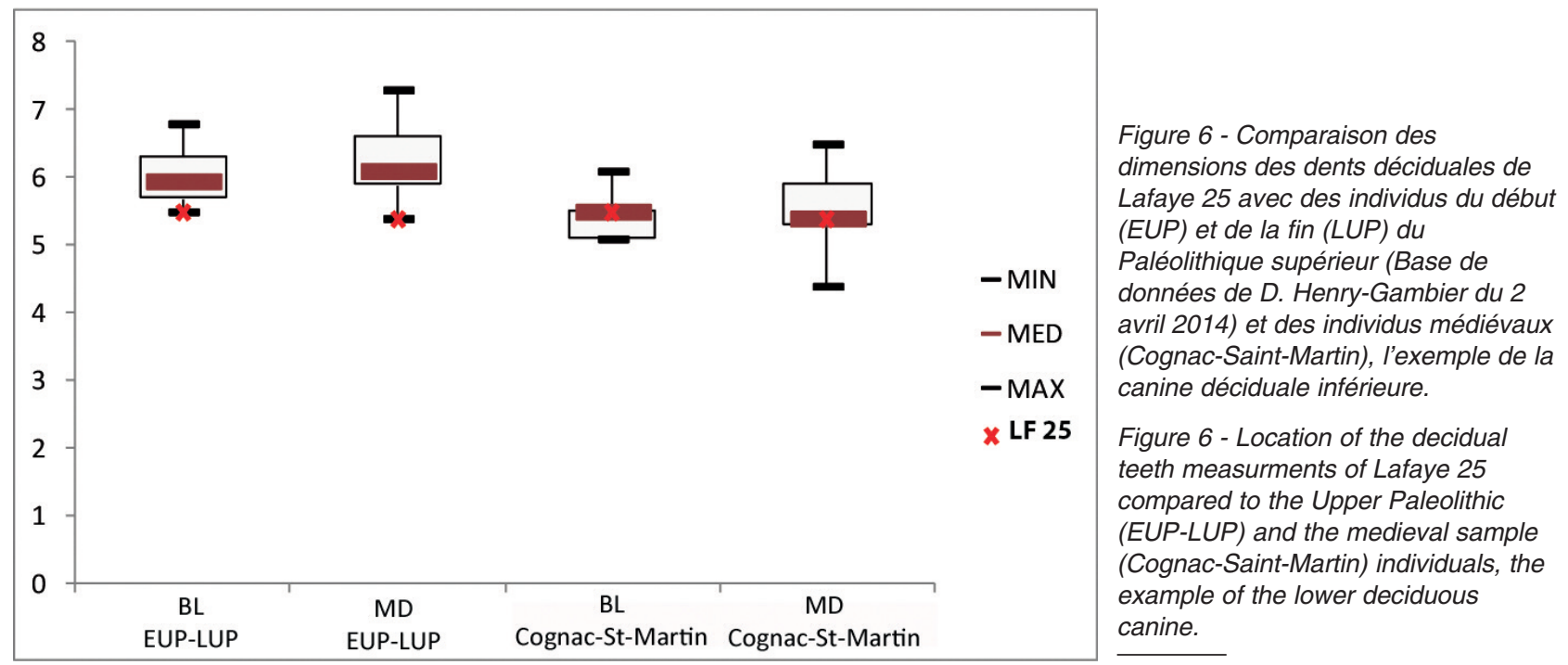

\section{Dents permanentes}

Les couronnes des germes des dents permanentes étant incomplètement calcifiées, leurs diamètres et en particulier le diamètre BL, sont sous-évalués. La comparaison avec l'adulte, Lafaye 24, qui présente des dents très usées, et les autres sujets du Paléolithique supérieur dont les couronnes ont atteint leur plein développement n'a donc guère de sens. On peut cependant noter que le diamètre MD du germe de la seconde incisive permanente supérieure de LF25 est très proche de celui de l'adulte et remarquer que les diamètres des incisives supérieures de LF24 se situent parmi les valeurs basses de la variation pour le Paléolithique supérieur (EUP-LUP).

La première molaire supérieure droite de LF25 offre un volume analogue à celui de la dent de LF24, ces deux individus se caractérisant par des diamètres $B L$ et $M D$ élevés comparés aux moyennes des échantillons du Paléolithique supérieur et se situant parmi les valeurs hautes de la variation (tabl. 4).

Les dimensions des dents permanentes présentent sur Lafaye 25 ont aussi été comparées à celles de l'échantillon médiéval. Les dimensions dentaires de notre sujet apparaissent plus volumineuses que celles des individus de la série du Moyen-Âge.

\section{5) Analyse morphologique comparative des dents}

La caractéristique morphologique à relever chez l'enfant de l'abri Lafaye est le nombre de cuspides surnuméraires sur les molaires déciduales et permanentes: tubercule de Carabelli (CAR), paracône mésial (MPT), protoconule (PL), métaconule (ML) ; hypoconulide déporté sur la face vestibulaire, entoconulide (fig. 7). En revanche, les molaires permanentes de l'adulte ne présentent pas de tubercules surnuméraires autant que leur fort degré d'usure ${ }^{8}$ permette d'en juger.

Ce type de variations affectant le nombre, la disposition et le degré d'expression de ces cuspides supplémentaires est connu dans les populations actuelles. Les études indiquent que les fréquences inter et intra populationnelles varient et interrogent sur les interactions entre le développement des cuspides supplémentaires et le processus de formation de la couronne dentaire. Parmi ces variations, le tubercule de Carabelli (CAR) sur les dents permanentes a fait l'objet de nombreux travaux (ex. Reid, van Reenen, Groeneveld 1991 ; Kondo et al. 2009 ; Ortiz et al. 2012 ; Townsend et al. 2012 ; Moormann, Guatelli-Steinberg, Hunter 2013). Ceuxci ont concerné le degré d'expression, la fréquence dans une population et son hérédité ainsi qu'une possible interaction (Moormann, Guatelli-Steinberg et Hunter 2013), entre le patrimoine génétique et le contexte environnemental. Pour Reid et collaborateurs (1991), la fréquence et le volume du tubercule de Carabelli seraient d'autant plus importants que la couronne serait large. Dérivé du cingulum, ce tubercule serait un trait archaïque. Ces hypothèses demanderaient à être confirmées par l'étude d'échantillons pertinents de dents de populations actuelles et notamment par celle d'échantillons de dents déciduales.

Dans le cas des populations fossiles du Paléolithique supérieur d'Europe, il est impossible de proposer une évaluation satisfaisante du type et du nombre de cuspides surnuméraires en raison notamment de la forte usure des couronnes et de la diversité des cotations selon les auteurs. En dépit de ces difficultés, deux remarques peuvent être faites : les cuspides surnuméraires sont loin d'être rares dans les échantillons du Paléolithique supérieur ; par exemple, la fréquence du tubercule de Carabelli sur les secondes molaires déciduales et les premières molaires

(8) La hauteur des couronnes est réduite de moitié. 

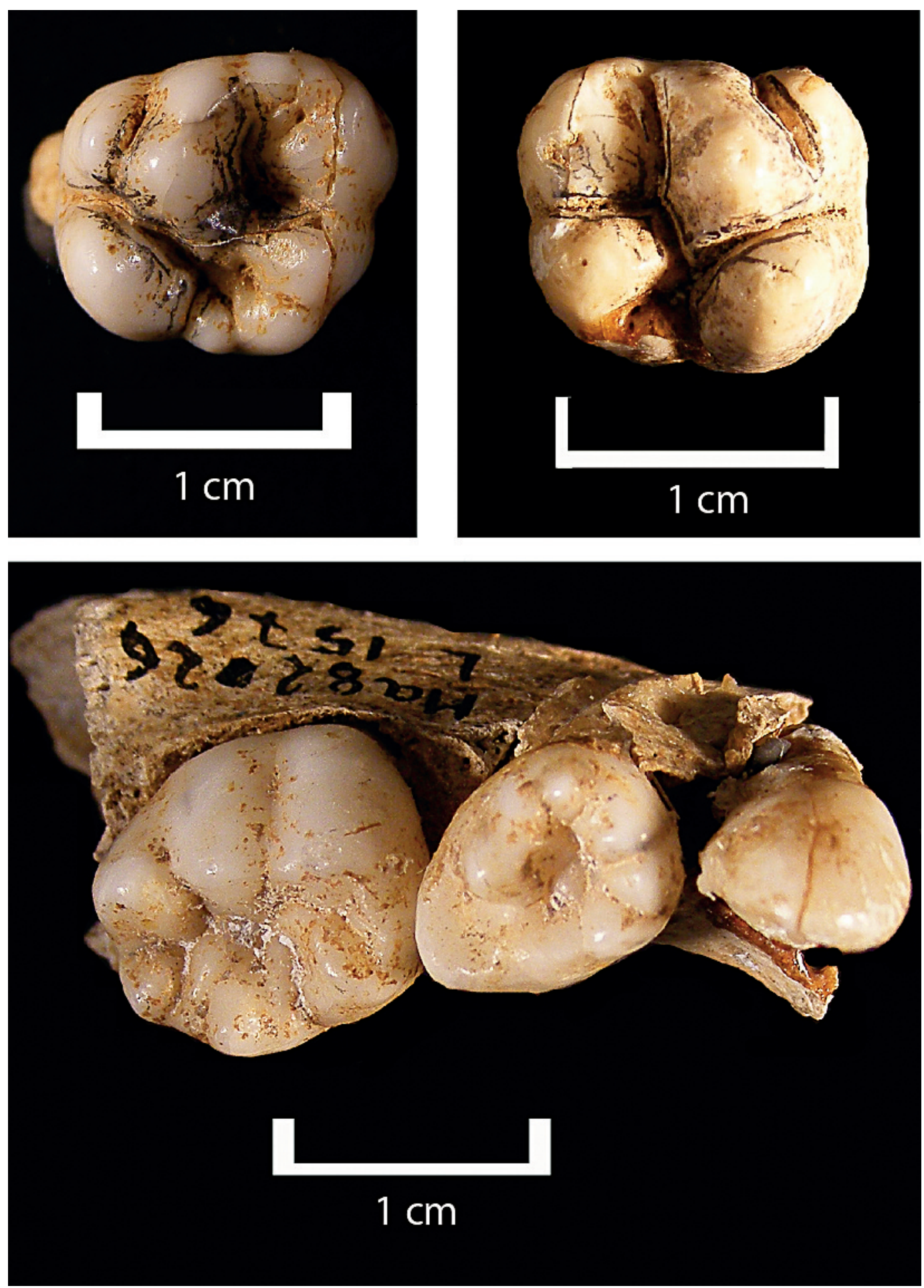

Figure 7 - Détails des cuspides surnuméraires de Lafaye 25.

Figure 7 - Non metric traits from teeth of Lafaye 25. 
permanentes de la fin du Paléolithique supérieur n'est pas négligeable (Henry-Gambier 2001). Deux des enfants de l'abri Pataud (Dents 48851 et 72 ) respectivement issus des couches gravettiennes (C4 et C2) présentent également un nombre élevé de tubercules supplémentaires. L'adulte féminin de la couche 2, P1 se caractérise aussi par des cuspides supplémentaires sur les dents permanentes supérieures (Legoux 1975 ; Henry-Gambier et al. 2013 ; Villotte et al. 2015). Les groupes Gravettiens en Moravie (Dolni Vestonice et Pavlov) en présentent également à différents stades d'expression sur les dents permanentes et déciduales (Hillson 1996) ; on en retrouve aussi dans les groupes plus tardifs, magdaléniens et épigravettiens (Henry-Gambier 2001).

Le développement du bord marginal sur la face linguale des incisives déciduales supérieures de Lafaye 25 (stade 1, Hanihara 1961) est classique sur beaucoup de dents déciduales du Paléolithique supérieur déterminant un " effet en pelle » peu marqué. Cet effet serait, selon certains auteurs, corrélé avec des dimensions importantes de la couronne dentaire. II pourrait être lié aux mécanismes de morphogénèse (temps et durée de développement, taille de l'arcade dentaire du maxillaire ; Trinkaus 2011) et ce caractère aurait un déterminisme génétique. L'usure des incisives supérieures de l'adulte, LF24, ne permet pas d'en discuter la morphologie. Hormis quelques exceptions - ex. les incisives centrales de l'adolescent gravettien de la sépulture double de la Grotte des Enfants en Ligurie, (Henry-Gambier, Maureille, White 2004), «l'effet en pelle» dans les populations du Paléolithique supérieur est observable mais à des fréquences et des niveaux d'expression très variables (Henry-Gambier, Maureille, White 2004 ; Trinkaus 2011).

\section{6) Analyse comparative du squelette}

L'état de conservation du squelette de Lafaye 25 limite les comparaisons métriques et morphologiques. Cependant quelques éléments osseux, tels que la Pars basilaris de l'os occipital, les clavicules et des os de la main sont mesurables (tabl. $2 ; 4$ et 5 ).

Ainsi la partie basilaire (fig.8) se démarque de l'échantillon médiéval par une longueur sagittale et une hauteur de synchondrose plus faible, alors que les autres dimensions sont plus élevées. Une forte variabilité des dimensions dans le groupe paléolithique existe.

Les clavicules dont la longueur n'est pas entièrement conservée sont graciles ce dont témoigne la valeur de la circonférence au foramen très faible (tabl. 5). En effet que ce soit en comparaison avec l'échantillon, fossile ou médiéval, les dimensions de LF25 se situent parmi les valeurs faibles de la variabilité, voir à l'extrême limite (fig. 9).

Les os de la main sont quant à eux exclusivement comparés à ceux de l'échantillon médiéval (tabl. 6 ; fig. 10). Le possible $3^{e}$ métacarpien de LF25 est plus court; la largeur s'intègre dans la variabilité. Le même constat peut être fait pour les phalanges proximales. Les phalanges médiales et distales quant à elles sont comparables.

In fine, comparé à celui d'enfants du Paléolithique supérieur et de populations récentes, le squelette infracrânien de Lafaye 25 ne présente pas de caractéristiques métriques et morphologiques spécifiques.

\section{Conclusions}

L'intérêt de cette étude réside dans la description de vestiges immatures inédits, contemporains du Magdalénien moyen : Lafaye 25 représente en effet l'un des rares enfants associés à cette culture (Gambier et Lenoir 1991 ; Henry-Gambier 2014).

Découvert il y a plus de 150 ans, l'état de conservation est malheureusement mauvais, et le squelette est très incomplet. La présence de petits os des mains (ex. phalanges distales), l'état de surface des os, le type de cassure indiquent que les fouilles, puis les aléas de la

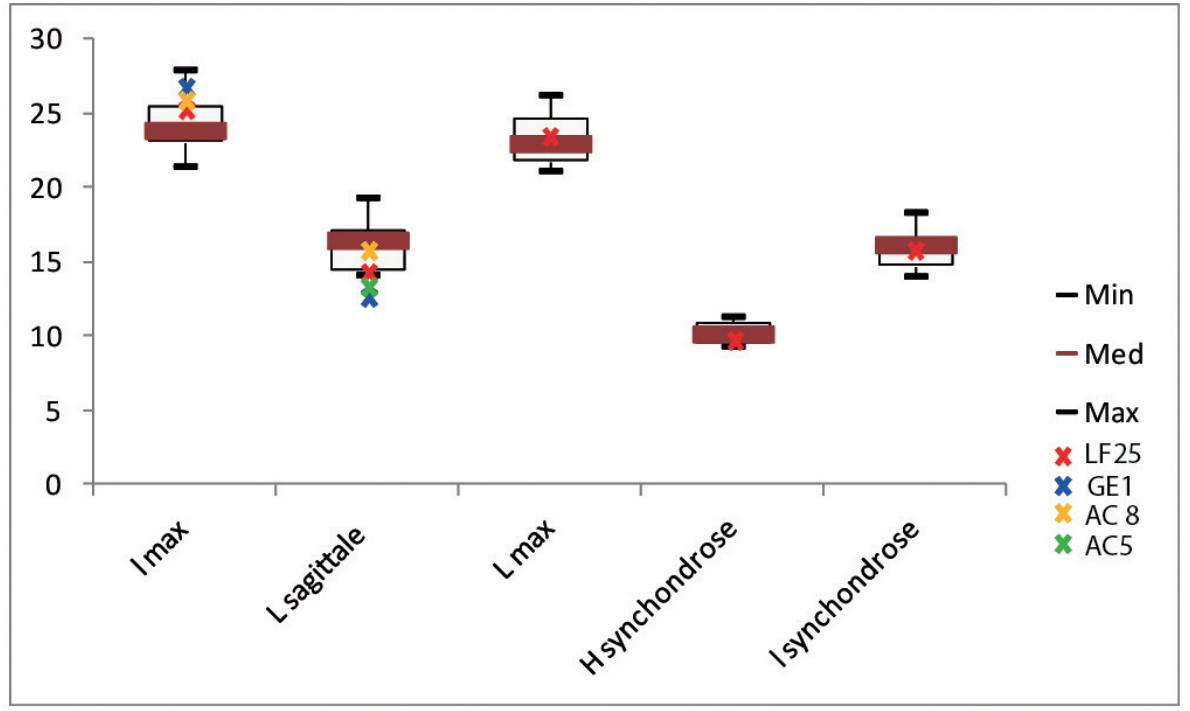

Figure 8 - Position des dimensions des parties basilaires de Lafaye 25 , Grotte des enfants 1, Arene Candide 8 et 5, (1): Henry-Gambier 2001, par rapport aux individus de l'échantillon médiéval.

Figure 8 - Location of the pars basilaris dimensions of Lafaye 25 , Grotte des enfants 1, Arene Candide 8 et 5 compared to the medieval sample individuals. 
Circonférence de la clavicule au foramen

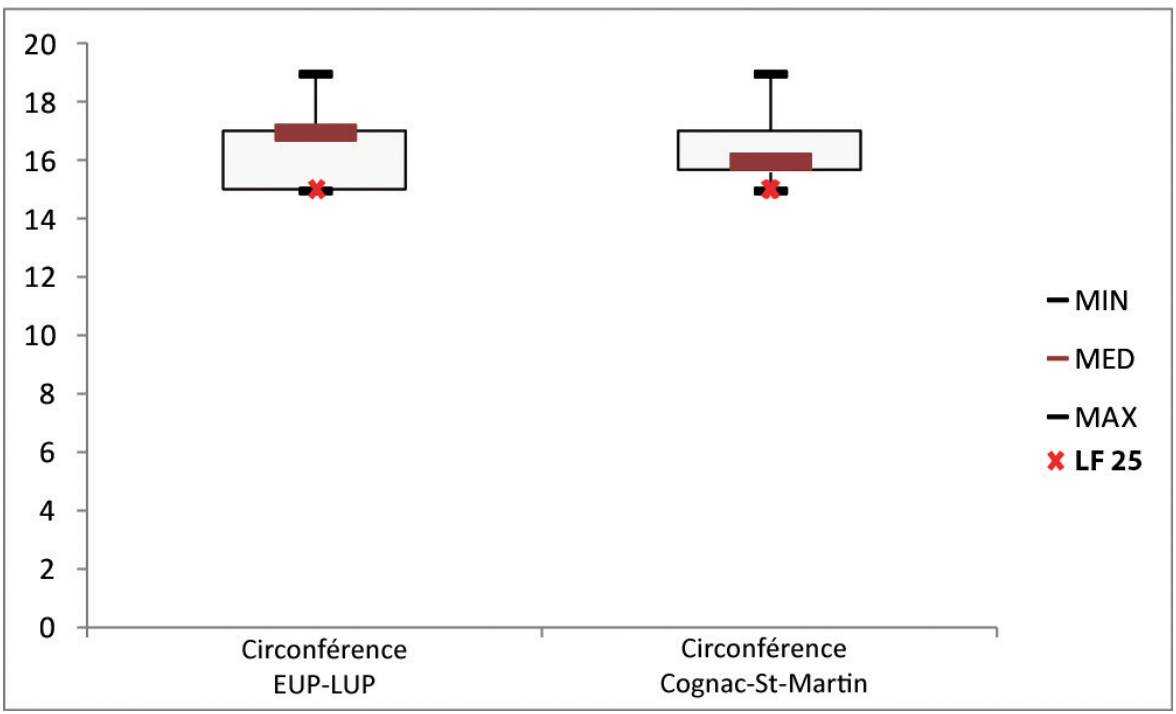

Figure 9 - Position des mesures des clavicules de Lafaye 25 par rapport aux individus du Paléolithique supérieur (EUP-LUP), (1) : HenryGambier 2001 et de l'échantillon médiéval (Cognac-St-Martin).

Figure 9 - Location of the clavicle measurements of Lafaye 25 compared to the Upper Paleolithic (EUP-LUP) and the medieval sample (Cognac-St-Martin) individuals.
Longueur et largeur des os de la main

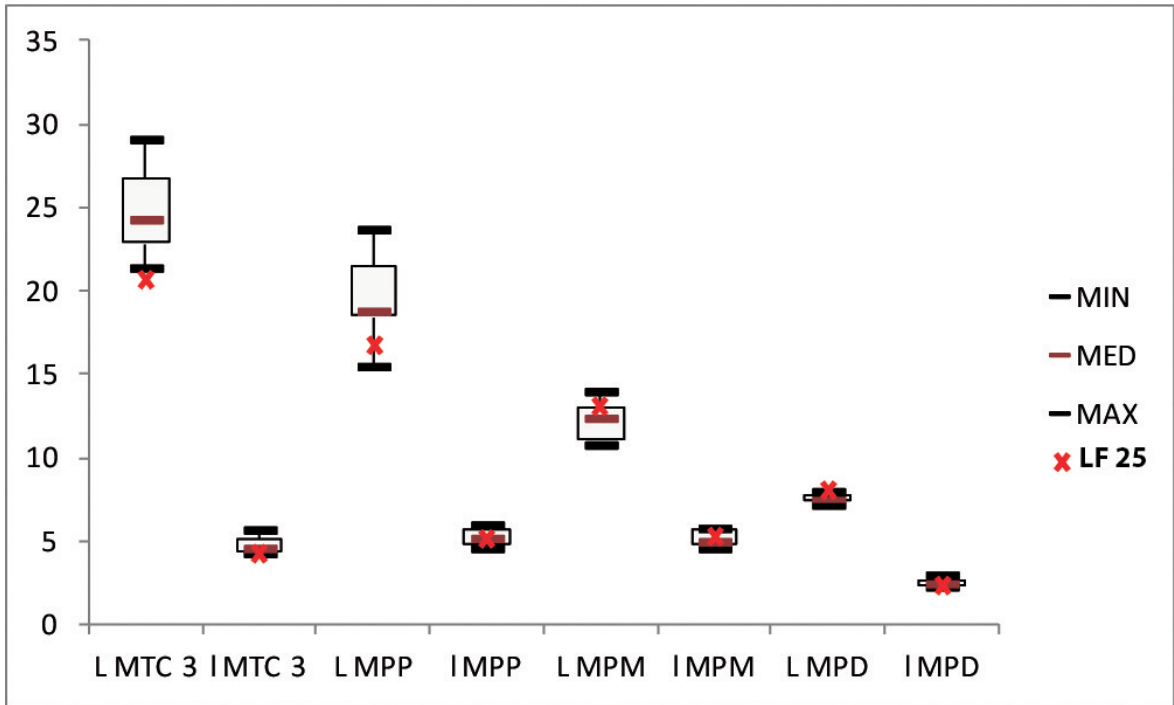

Figure 10 - Position des longueurs et largeurs des os de la main de Lafaye 25 par rapport aux individus de l'échantillon médiéval (CognacSaint-Martin).

Figure 10 - Location of length and width of the bones of the hand of Lafaye 25 compared to medieval sample individuals (CognacSaint-Martin). conservation et de la restauration sont les grands responsables de cette situation. L'état des os collectés et conservés suppose un enfouissement comme ceux de l'adulte féminin avec qui il avait été déposé.

La fragmentation des os et des dents limite l'information biologique et les comparaisons que ce soit avec des populations actuelles, archéologiques ou fossiles.

Les analyses des dents et de la maturation osseuse indiquent un sujet dont l'âge se situe entre 1 et 4 ans et plus probablement dans la moitié supérieure de cet intervalle. II ne présente aucun caractère métrique ou morphologique particulier comparé à des enfants fossiles contemporains ou d'époque médiévale d'anatomie moderne et d'âge comparable. La présence de cuspides surnuméraires constitue un des seuls traits notables.
Les causes de la mort, comme cela est généralement le cas, ne sont pas connues. Aucune lésion d'ordre pathologique n'a été observée. Seule la présence d'hypoplasies interpelle sur l'état de santé « précaire » en fin de vie de Lafaye 25. Toujours dans le registre dentaire, rappelons la présence d'un tubercule de Carabelli très développé sur les secondes molaires déciduales et les premières molaires permanentes. Ce trait, connu chez divers adultes et enfants du Paléolithique supérieur est mentionné par certains comme un indice de cause de mortalité accrue (Murail 1996 ; Bocquentin 2003) mais une étude approfondie de la fréquence de ce caractère est nécessaire pour valider cette hypothèse. La question du lien de parenté avec la femme adulte avec laquelle il gisait, se pose. On ne retrouve pas chez l'adulte les cuspides 
surnuméraires ${ }^{9}$, et notamment le tubercule de Carabelli, Le crâne de l'individu adulte ne présente pas de suture pétrosquameuse, alors que cette dernière est visible chez l'enfant. Ces données sont très pauvres pour défendre ou réfuter un éventuel lien de parenté entre ces deux sujets, longtemps considérés comme une mère et son enfant (Guéret 1953). L'âge de la femme (20-49 ans ; Villotte 2009), rend plausible cette hypothèse mais ne l'étaye pas.

\section{Remerciements}

Nous tenons à remercier E. Ladier qui en tant que conservatrice du Museum d'Histoire naturelle de Montauban nous a confié l'étude de l'enfant de l'abri Lafaye. Nos remerciements vont également à $\mathrm{C}$. Beauval (Archéosphère) pour les discussions sur l'état de conservation des os, ainsi qu'à Anne-marie Tillier pour sa relecture et ses conseils. Ils s'adressent aussi deux relecteurs pour leur lecture attentive et leurs remarques constructives.

\section{Références bibliographiques}

BLAKEY M.L. et ARMELAGOS G.J. 1985 - Deciduous enamel defects in Prehistoric Americans from Dickson Mounts: Prenatal and postnatal stress. Am. J. Phys. Anthropol., 66, p. 371-380.

BLAKEY M.L. et ARMELAGOS G.J. 1997 - Comment on " Hypoplastic Area Method for Analyzing Enamel Hypoplasia ». Am. J. Phys. Anthropol., 102, p. 295-299.

BOCQUENTIN F. 2003 - Pratiques funéraires, paramètres biologiques et identités culturelles au Natoufien : une analyse archéo-anthropologique. Bordeaux : Université Bordeaux 1, 2003. 629 p. Thèse de Doctorat en Anthropologie Biologique : Bordeaux 1.

BRAÜER G. 1988 - Osteometrie. In : R., Knussmann, R. Martin (Eds.), Anthropologie. Handbuch der vergleichenden Biologie des Menschen. Fischer, Stuttgart, p. 160-232.

BROCA P. 1866 - Sur les fouilles pratiquées par M. Brun dans la caverne-abri de Bruniquel. BMSAP, 1, p. 48-52.

BRUN V. 1866 - Exploitation de la caverne Abri de Lafaye à Bruniquel, Lettre manuscrite à $\mathrm{E}$. Lartet, Montauban, le 15 Janvier 1866

DUDAY H., LAUBENHEIMER F., TILLIER A.-m. 1995 Nouveau-nés et nourrissons gallo-romains. Centre de Recherches d'Histoire Ancienne, 144, CNRS, série amphores, 3, Paris.

FAZEKAS I. et KOSA F. 1978 - Forensic foetal osteology. Akademiai Kiado, Budapest.
GAMBIER D. 1992 - Les populations magdaléniennes en France. In : J.P. Rigaud, H. Laville, B. Vandermeersch (Eds.), Le peuplement magdalénien : Paléogéographie physique et humaine. C.T.H.S., Paris, pp. 41-51.

GAMBIER D., LENOIR M. 1991. Les vestiges humains du Paleolithique supérieur en Gironde. Bull. de la Société d'Anthropologie du Sud-Ouest, 26, 1, p. 1-31.

GAMBIER D., VALLADAS H., TISNERAT-LABORDE N., ARNOLD M., BRESSON F. 2000 - Datation de vestiges humains présumés du Paléolithique supérieur par la méthode du Carbone 14 en spectrométrie de masse par accélérateur. Paleo, 12, p. 201-212.

GENET-VARCIN E. et MIQUEL M. 1967 - Contribution à l'étude du squelette magdalénien de l'abri Lafaye à Bruniquel (Tarn et Garonne). L'Anthropologie, 7, 5-6, p. 467-478.

GOODMAN A.H., ARMELAGOS G.J., ROSE J.C. 1984 The Chronological Distribution of Enamel Hypoplasias From Prehistoric Dickson Mounds Populations. Am. J. Phys. Anthropol., 65, p. 259-266.

GOODMAN A.H. et ROSE J.C. 1990 - Assessment of systemic physiological perturbations from dental enamel hypoplasias and associated histological structures. Am. J. Phys. Anthropol., 33, p. 59-110.

GUATELLI-STEINBERG D., BUZHILOVA A.P., TRINKAUS E. 2011 - Developmental stress and survival among the Mid Upper Paleolithic Sunghir children: Dental Enamel Hypoplasias of Sunghir 2 and 3 . Int. J. Osteoarchaeol. 10.1002/oa.1263.

GUÉRET M. 1953 - Les squelettes magdaléniens du Musée de Montauban. Bulletin Archéologique du Comité des Travaux historiques et scientifiques, p. 151-169.

HAMY E.T. 1874 - Description d'un squelette humain fossile de Laugerie-Basse. BMSAP, 9, p. 652-658.

HANIHARA K. 1961 - Criteria for Classification of Crown Characters of the Human Deciduous Dentition. J. of the Anthropological Society of Nippon, 69, p. 27-45.

HENRY-GAMBIER D. 2001 - La sépulture des enfants de Grimaldi (Baoussé-Roussé, Italie) Anthropologie et palethnologie funéraire des populations de la fin $d u$ Paléolithique supérieur, CTHS, Paris.

HENRY-GAMBIER D., MAUREILLE B., WHITE R. 2004 Vestiges humains des niveaux de l'Aurignacien ancien du site de Brassempouy (Landes). BMSAP, Paris, 16, 1-2, p. 49-87. 
HENRY-GAMBIER D. 2006 - Les sépultures de Sorde l'Abbaye (Landes). - In : M. Dachary (Ed.), Les Magdaléniens à Duruthy. Qui étaient-ils ? Comment vivaient-ils ? Centre Départemental du Patrimoine, Hastingues, octobre 2006, p. 67-73.

HENRY-GAMBIER D. 2014 - Les pratiques funéraires magdaléniennes en Europe. Grands sites d'art magdalénien. La Madeleine et Laugerie-Basse il y a 15000 ans, Paris : Ed. RMN, p 55-57.

HENRY-GAMBIER D., BRUZEK J., SCHMITT A., HOUËT F., MURAIL P. 2007 - Changement de paradigme dans la détermination du sexe et de l'âge au décès des sujets adultes à partir du squelette : application aux fossiles du Paléolithique supérieur d'Europe. In : J. Evin (Ed.), Un siècle de construction du discours scientifique en Préhistoire. Actes du XXVlè Congrès Préhistorique de France, Avignon, 20-25 septembre 2004, volume III., Paris : Société préhistorique française, p. 515-525.

HENRY-GAMBIER D., VILLOTTE S., BEAUVAL C., BRUZEK J., GRIMAUD-HERVÉ D. 2013 - Les vestiges humains: un assemblage original. In : R. Nespoulet, L. Chiotti et D. Henry-Gambier (Eds), Le Gravettien Final de L'abri Pataud (Dordogne, France) Fouilles et Études 2005e2009, BAR International Series 2458, p. 135-178.

HILLSON S. 1996 - Dental anthropology. Cambridge University Press. 393p.

HILLSON S. et BOND S. 1997 - Relationship of enamel hypoplasia to the pattern of tooth crown growth : a discussion. Am. J. Phys. Anthropol., 104, p. 89-103.

HOLCOMB S. et KONIGSBERG L. 1995 - Statistical study of sexual dimorphism in the human fetal sciatic notch. Am. J. Phys. Anthropol., 97, p. 113-125.

KONDO T., HANEJI K., HANIHARA T., MATSUKUSA H., KAWAMURA S., MAKI K., OSAWA M., ISHIDA H., OOTA H. 2009 - A Common Variation in EDAR Is a Genetic Determinant of Shovel-Shaped Incisors. The Am. J. of Human Genetics, 85, p. 528-535.

LADIER E. et WELTÉ A. C. 1991 - La vallée de L'Aveyron, De Bruniquel à Fontalès : nouvelles observations. Bull. de la Société Méridionale de Spéléologie et de Préhistoire, 31, p. 51-76.

LEGOUX P. 1975 - Présentation des dents des restes humains de l'Abri Pataud. In: H.L. Movius (Ed), Excavation of the Abri Pataud. Harvard Univ. Cambridge Mass, p. 262-305.

LOTH S. et HENNEBERG M. 2001 - Sexually Dimorphic Mandibular Morphology in the First Few Years of Life. Am. J. Phys. Anthropol., 115, p. 179-186.

MAJÒ T., TILLIER A.-m., BRUZEK J. 1993 - Test des fonctions discriminantes de Schutkowski impliquant l'ilium pour la détermination du sexe dans des séries d'âge et de sexe connus. BMSAP, 5, 1-2, p. 61-68.
MALVILLE N. J. 1997 - Enamel Hypoplasia in Ancestral Puebloan Populations From Southwestern Colorado: I. Permanent Dentition. Am. J. Phys. Anthropol., 102, p. 351-367.

MOLLESON T. et CRUSE K. 1998 - Some sexually dimorphism features of the Human juvenile skull and their value in sex determination in immature skeletal remains. $J$. Archaeol. Sci., 25, p; 719-728.

MOORMANN S., GUATELLI-STEINBERG D., HUNTER J. 2013 - Metamerism, Morphogenesis, and the expression of Carabelli and other dental traits in Humans. Am. J. Phys. Anthropol. DOI 10.1002/ajpa.22216.

MOORREES C., FANNING E., HUNT E. 1963a - Age variation of formation stages for ten permanent teeth. Journal of Dental Research, 42, p. 1490-1502.

MOORREES C., FANNING E., HUNT E. 1963b Formation and resorption of three deciduous teeth in children. Am. J. Phys. Anthropol., 21, p. 205-213.

MURAIL P. 1996 - Biologie et pratiques funéraires d'époque historique : une démarche méthodologique appliquée à la nécropole gallo-romaine de Chantambre (Essonne, France). Bordeaux : Université Bordeaux 1, 1996. 264 p. Thèse de doctorat en Anthropologie : Bordeaux 1.

OAKLEY K.P., CAMPBELL B.G., MOLLESON T. 1971 Catalog of fossil Hominids. Part II : Europe. British Museum (Natural History) of London, 379 p.

ORTIZ A., SKINNER M., BAILEY S., HUBLIN J.J. 2012 Carabelli's trait revisited: An examination of mesiolingual features at the enamel-dentine junction and enamel surface of Pan and Homo sapiens upper molars. J. Hum. Evol,. 63, p. 586-596.

REID C., van REENEN J.F., GROENEVELD H.T. 1991 Tooth size and the Carabelli Trait. Am. J. Phys. Anthropol., 84 , p. 427-432.

REID D.J. et DEAN M.C. 2000 - Brief communication: The timing of linear hypoplasias on Human anterior teeth. Am. J. Phys. Anthropol., 113, p. 135-139.

REIMER P.J., BARD E., BAYLISS A., BECK J.W., BLACKWELL P.G., BRONK RAMSEY C., BUCK C.E., CHENG H., EDWARDS R.L., FRIEDRICH M., GROOTES P.M., GUILDERSON T.P., HAFLIDASON H., HAJDAS I., HATTÉ C., HEATON T.J., HOGG A.G., HUGHEN K.A., KAISER K.F., KROMER B., MANNING S.W., NIU M., REIMER R.W., RICHARDS D.A., SCOTT E.M., SOUTHON J.R., TURNEY C.S.M., van der PLICHT J. 2013 - IntCal13 and MARINE13 radiocarbon age calibration curves 0-50000 years calBP \# Radiocarbon, 55(4).

SANSILBANO-COLLILIEUX M. 1993 - Note sur la discordance entre âge dentaire, âge osseux et âge statural : l'exemple des sujets immatures de la nécropole 
médiévale de Saint-Martin de Cognac. BMSAP, 5, 1-2, p. 103-108.

SAUNDERS S.R., CHAN A.H.W., KAHLON B., KLUGE H.F., FITZGERALD C.M. 2007 - Sexual dimorphism of the dental tissues in human permanent mandibular canines and third premolars. Am. J. Phys Anthropol., 133, p. 735-740.

SCHEUER L. et BLACK S. 2000 - Developemental of juvenile osteology. Academic Press, London.

SCOTT G.R. et TURNER C.G. 1997 - Description and classification of permanent crown and root traits. In : G.R. Scott et C.G. Turner (Eds.), The anthropology of modern human teeth. Dental morphology and its variation in recent human populations, Cambridge University Press, p. $15-69$.

SILBERMAN H. 1989 - Consequences of malnutrition. In : H. Siberman (Ed), Parental and Enteral Nutrition, Appleton \& Lange, Norwalk, p. 1-18.

SKINNER M. F. 1996 - Developmental stress in immature Hominines from Late Pleistocene Eurasia : Evidence from enamel hypoplasia. J. Archaeol. Sci., 23, p. 833-852.

SKINNER M. F. et GOODMAN A. H. 1992 Anthropological Uses of Developmental Defects of Enamel. In : S. R. Saunders, M. A. Katzenberg (Eds), Skeletal Biology of Past Peoples: Research Methods, Wiley-Liss, New York, p. 153-174.

SKINNER M. F. et HUNG J. T. W. 1989 - Social and biological correlates of localized enamel hypoplasia of the human deciduous canine tooth. Am. J. Phys. Anthropol., 79,2 , p. $159-175$.

SLADEK V., TRINKAUS E., HILLSON S.W., HOLLIDAY T.W. 2000 - The people of the Pavlovian: Skeletal catalogue and osteometrics of the Gravettian fossil hominids from Dolni Vestonice and Pavlov. Academy of Science of the Czech Republic Brno, p. 244.
SMITH B.H. 1984 - Patterns of molar wear in huntergatherers and agriculturists. Am. J. Phys. Anthropol., 63, p. 39-56.

TOWNSEND G., BOCKMANN M., HUGHES T., BROOK A. 2012 - Genetic, environmental and epigenetic influences on variation in human tooth number, size and shape. Odontology, 100, p. 1-9.

TRINKAUS E. 2011 - Late Neandertals and early modern Humans in Europe, population dynamics and paleobiology. In: S. Condemi et G.-C. Weniger (Eds), Continuity and discontinuity in the peopling of Europe: One hundred fifty years of Neanderthal study. Springer, Vertebrate Paleobiology and Paleoanthropology Series.

UBELAKER D.H. 1978 - Human skeletal remains: excavations, analysis, interpretation. Taraxacum, Washington.

VILLOTTE S. 2009 -. Enthésopathies et activités des hommes préhistoriques. Recherche méthodologique et application aux fossiles européens du Paléolithique supérieur et du Mésolithique, (BAR International Series 1992). Oxford: Archaeopress, 205 p.

VILLOTTE S., CHIOTTI L., NESPOULET R., HENRYGAMBIER D. 2015 - Étude anthropologique des vestiges humains récemment découverts issus de la couche 2 de l'abri Pataud (Les Eyzies-de-Tayac-Sireuil, Dordogne, France). BMSAP, 27(3-4), p. 158-188.

WELLS C. 1967 - A new approach to paleopathology: Harris's lines. Diseases in Antiquity, 30, p. 390-404. 